\title{
Ultra-cool dwarfs: new discoveries, proper motions, and improved spectral typing from SDSS and 2MASS photometric colors ${ }^{\star}$
}

\author{
Z. H. Zhang ${ }^{1,3,2}$, R. S. Pokorny ${ }^{1}$, H. R. A. Jones ${ }^{2}$, D. J. Pinfield ${ }^{2}$, P. S. Chen ${ }^{1}$, Z. Han ${ }^{1}$, D. Chen ${ }^{1,2}$, \\ M. C. Gálvez-Ortiz ${ }^{2}$, and B. Burningham ${ }^{2}$
}

\author{
1 National Astronomical Observatories/Yunnan Observatory, Chinese Academy of Sciences, Kunming 650011, PR China \\ e-mail: zenghuazhang@hotmail.com \\ 2 Centre for Astrophysics Research, Science and Technology Research Institute, University of Hertfordshire, College Lane, \\ Hatfield AL10 9AB, UK \\ e-mail: h.r.a.jones@herts.ac.uk \\ 3 Graduate School of Chinese Academy of Sciences, Beijing 100049, PR China
}

Received 3 June 2008 / Accepted 14 January 2009

\section{ABSTRACT}

\begin{abstract}
Aims. We try to identify ultra-cool dwarfs from the seventh Data Release of the Sloan Digital Sky Survey (SDSS DR7) with SDSS $i-z$ and $r-z$ colors. We also obtain proper motion data from SDSS, 2MASS, and UKIDSS and improve spectral typing from SDSS and 2MASS photometric colors.

Methods. We selected ultra-cool dwarf candidates from the SDSS DR7 with new photometric selection criteria, which are based on a parameterization study of known L and T dwarfs. The objects are then cross-identified with the Two Micron All Sky Survey and the Fourth Data Release of the UKIRT Infrared Deep Sky Survey (UKIDSS DR4). We derive proper motion constraints by combining SDSS, 2MASS, and UKIDSS positional information. In this way we are able to assess, to some extent, the credence of our sample using a multi epoch approach, which complements spectroscopic confirmation. Some of the proper motions are affected by short baselines, but, as a general tool, this method offers great potential to confirm faint L dwarfs as UKIDSS coverage increases. In addition we derive updated color-spectral type relations for L and T dwarfs with SDSS and 2MASS magnitudes.

Results. We present 59 new nearby M and L dwarfs selected from the imaging catalog of the SDSS DR7, including proper motions and spectral types calculated from the updated color-spectral type relations. and obtain proper motions from SDSS, 2MASS, and UKIDSS for all of our objects.
\end{abstract}

Key words. stars: low-mass, brown dwarfs - stars: late-type

\section{Introduction}

Brown dwarfs occupy the mass range between the lowest mass stars and the highest mass planets. The central temperature of a brown dwarf is not high enough to achieve stable hydrogen burning like a star, but all brown dwarfs will undergo short periods of primordial deuterium burning very early in their evolution. Since the first discovery of an L dwarf (GD165 B; Becklin \& Zuckerman 1988) and a T dwarf (G1229 B; Nakajima et al. 1995), the projects searching for brown dwarfs have involved a number of large scale surveys, for example, the Deep Near-Infrared Survey (DENIS; Epchtein et al. 1997), the Two Micron All Sky Survey (2MASS; Skrutskie et al. 2006) and the Sloan Digital Sky Survey (SDSS; York et al. 2000; AdelmanMaCarthy et al. 2008). $554 \mathrm{~L}$ dwarfs and $145 \mathrm{~T}$ dwarfs have been found in large scale sky surveys in the last decade (by January 2009, see, DwarfsArchives.org for a full list). 31 of L or T dwarfs have been found in DENIS (e.g. Delfosse et al. 1997), 185 in SDSS (e.g. Fan et al. 2000; Geballe et al. 2002; Hawley et al. 2002; Schneider et al. 2002; Knapp et al. 2004; Chiu et al. 2006), and 368 in 2MASS (e.g. Burgasser et al. 1999, 2002, 2004; Kirkpatrick et al. 1999, 2000; Gizis et al. 2000; Cruz et al. 2003, 2007; Kendall et al. 2003, 2007; Looper et al. 2007).

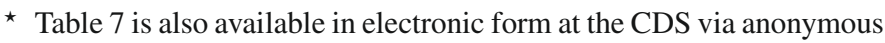
ftp to cdsarc.u-strasbg. fr $(130.79 .128 .5)$ or via http://cdsweb.u-strasbg.fr/cgi-bin/qcat?J/A+A/497/619
More recently, the UKIRT Infrared Deep Sky Survey (UKIDSS; Lawrence et al. 2007) is beginning to be very effective in searching for T dwarfs (Kendall et al. 2007; Lodieu et al. 2007; Warren et al. 2007; Burningham et al. 2008; Pinfield et al. 2008).

In this paper we report the discovery of 59 late $M$ and L dwarfs from the main photometric catalog of SDSS DR7.1. The photometric selection processes are presented in Sect. 2. The red optical spectra of the 36 new late $M$ and $L$ dwarfs from SDSS are presented in Sect. 3. Polynomial fitting for colorspectral type relationships are derived in Sect. 4. The UKIDSS matches for 23 ultra-cool dwarf candidates without SDSS spectra are presented in Sect. 5, and Sect. 6 presents some further discussion.

\section{Photometric selection}

The Sloan Digital Sky Survey uses a dedicated $2.5 \mathrm{~m}$ telescope located at Apache Point Observatory (APO) in New Mexico. It is equipped with a large format mosaic CCD camera to image the sky in five optical bands $(u, g, r, i, z)$, and two digital spectrographs to obtain the spectra of galaxies, quasars and late type stars selected from the imaging data (York et al. 2000). The SDSS DR7 imaging data covers about $8420 \mathrm{deg}^{2}$ of the main survey area (legacy sky), with information on roughly 230 million distinct photometric objects. The SDSS magnitude limits 

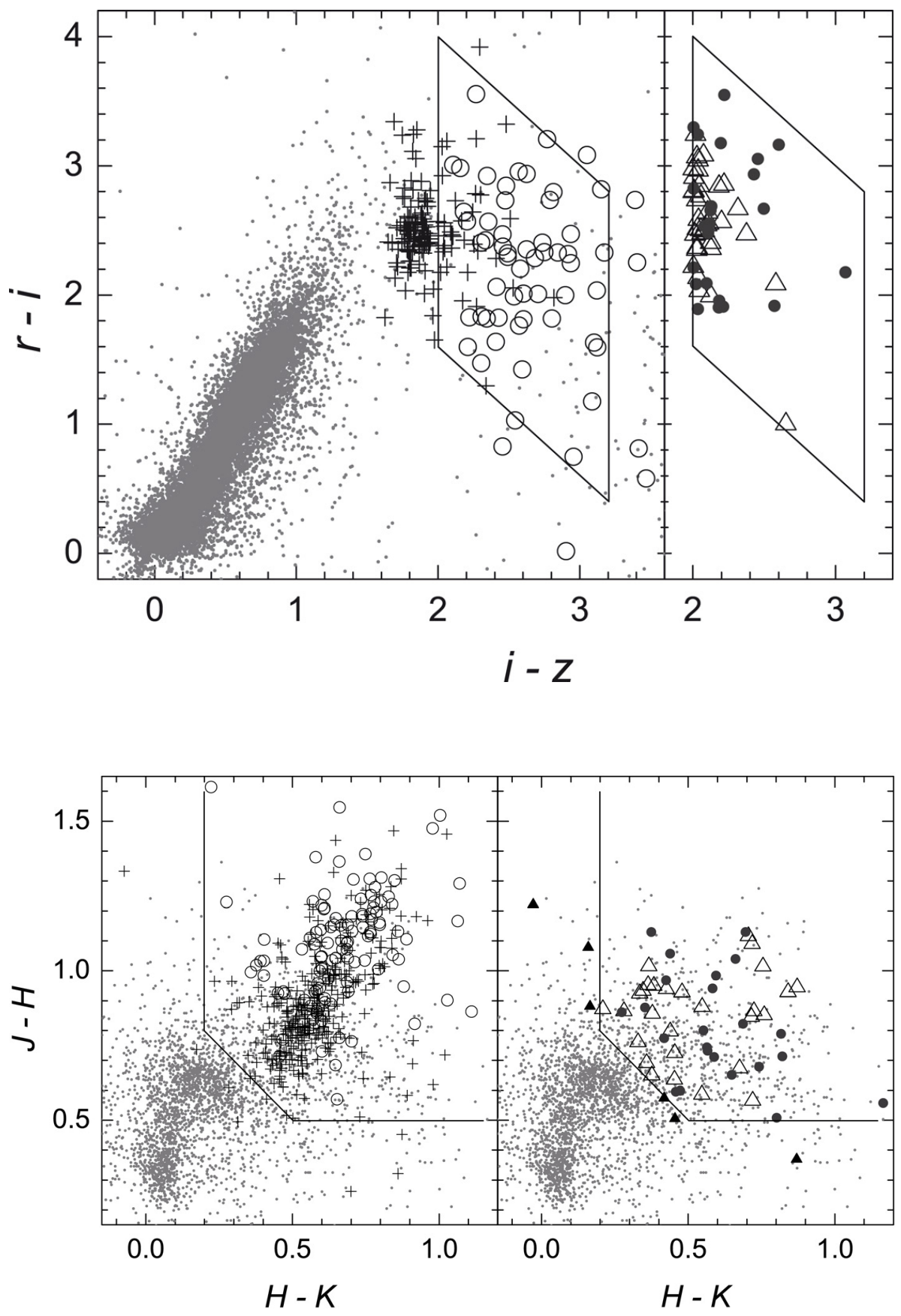

Fig. 1. $r-i$ vs. $i-z$ diagram for previously identified L dwarfs (left hand panel) and new M and $\mathrm{L}$ dwarfs (right hand panel). Previously identified L0-L4.5 and L5-L9.5 dwarfs are shown as crosses and open circles respectively. The 36 new $M$ and $L$ dwarfs with measured spectral types (from SDSS spectroscopy) are shown as open triangles, and the 23 new ultracool dwarf candidates (no SDSS spectra available) that we have cross-matched in UKIDSS DR4 are shown as filled circles. As a comparison, 24300 point sources with $15<z<20.5$ from $10 \mathrm{deg}^{2}$ of SDSS coverage are shown as dots. A parallelogram shows the boundary limits of our color selection.
Fig. 2. $J-H$ vs. $H-K$ diagram for previously identified L dwarfs (left hand panel) and new M and $\mathrm{L}$ dwarfs (right hand panel). Symbols are as in Fig. 1 except that six of the new spectroscopically confirmed dwarfs (three M9, one L0 and two L1) are indicated with filled triangles, because they lie outside our 2MASS photometric selection criteria (solid lines). Note that the 2MASS criteria are only applied when selecting photometric candidates, and not when SDSS spectra are available. For comparison, the plot shows 2800 sources (dots) taken from $3.14 \mathrm{deg}^{2}$ of 2 MASS sky.
(95\% detection repeatability for point sources) for the $u, g, r, i$ and $z$ bands are 22.0, 22.2, 22.2, 21.3 and 20.5 respectively.

The $i-z$ color is particularly useful for $\mathrm{L}$ dwarf selection (as first pioneered by Fan et al. (2000), and expanded on by others e.g. via the $i$-band drop-out method; e.g. Chiu et al. 2006). For the cooler $T_{\text {eff }} \mathrm{T}$ dwarfs, almost all of the radiation is emitted beyond $10000 \AA$, and as such these objects are optically much fainter than L dwarfs. SDSS is thus significantly less sensitive to $\mathrm{T}$ dwarfs than to $\mathrm{L}$ dwarfs, but has the sensitivity to identify $\mathrm{L}$ dwarfs out to distances well beyond $100 \mathrm{pc}$.

We have made a study of L dwarf color-color parameterspace using previously identified L and T dwarfs with photometric data available from either SDSS or 2MASS (from DwarfsArchives.org, as of September 25, 2007). Where two spectral types are available (optical and infrared) we used the mean average type. A total of $431 \mathrm{~L}$ and $84 \mathrm{~T}$ dwarfs have
2MASS photometric data $(J, H, K)$, and $193 \mathrm{~L}$ and $46 \mathrm{~T}$ dwarfs have SDSS photometric data $(u, g, r, i, z)$. We excluded $\mathrm{L}$ and $\mathrm{T}$ dwarfs known to be unresolved binary systems from our study. These optical and near-infrared parameter spaces are shown in the left-hand panels of Figs. 1 and 2, where crosses indicate early L dwarfs (L0-L4.5) and open circles indicate mid-late L dwarfs (L5-L9.5). Using these plots we have identified regions of color space that contain the vast majority of mid-late L dwarfs. We chose $i-z>2$ to avoid too much contamination from red dwarfs, although this does leads to missing many early $\mathrm{L}$ dwarfs. Figure 1 shows the selection cuts in the $r-i$ versus $i-z$ diagram, in which a parallelogram shows the boundary limits. Two two sloping lines show the boundary limits $r-z=3.6$ and $r-z=6$, and the two vertical lines show $i-z=2$ and $i-z=3.2$. Taking into account also the photometric sensitivities of SDSS, we thus define a set of SDSS mid-late L dwarf photometric selection 
criteria as follows:

$$
\begin{aligned}
& 19<i<23 \\
& 17<z<20 \\
& 3.6<r-z<6 \\
& 2<i-z<3.2
\end{aligned}
$$

Criterion (3) can also be written as

$3.6-(i-z)<r-i<6-(i-z)$

in $r i z$ color-color space.

The left-hand panel of Fig. 2 shows the $J-H$ vs. $H-K$ diagram for known L dwarfs. As for Fig. 1 we define a set of color criteria to contain the majority of these L dwarfs. It is clear from Fig. 2 that the L dwarfs appear reasonably well separated from the $\mathrm{M}$ dwarfs in this 2-color space (see also Burgasser et al. 2002), and we would thus expect to improve our sample refinement significantly by combining our optical selection with additional near infrared photometry. Our chosen 2MASS color selection criteria are shown in the figure with solid lines, and are defined as:

$J-H>0.5$
$H-K>0.2$
$J-K>1$.

More than 6000 candidates survived our color and magnitude optical selection from the main photometric catalog of SDSS DR7. Those candidates were matched with point sources in the 2MASS catalog (Skrutskie et al. 2006). We used a matching radius of 6 " to ensure that any ultra-cool dwarfs with high proper motion could be matched, despite possible motion over a period of up to $\sim 8$ years (between epochs). A total of 700 SDSS objects were cross-matched in 2MASS. Because the imaging depth of SDSS is deeper than that of 2MASS, many of the fainter SDSS candidates could not be found in 2MASS. Thus some of the candidates get wrongly matched with their nearest brighter neighbors in 2MASS. These are removed by checking their images in both the SDSS and 2MASS databases. On closer inspection, 2MASS mis-matches are usually very apparent, since the mis-matching objects generally have their own SDSS counterpart that immediately rules them out as high proper motion. As a more subtle check of the cross-matching, we assessed the spectral energy distribution of candidates (estimated from their SDSS and 2MASS photometry), and identified candidates that appeared to have unusual SEDs when compared to those of known ultra-cool dwarfs. It was assumed that these objects were also 2MASS mis-matches, and they were removed from our selection. Tens of wrongly matched objects were thus identified and removed from our selection. Implementation of our near infrared color selection criteria (for candidates that did not have SDSS spectroscopy) allowed us to remove more than 300 objects from our sample via the $J-H$ and $H-K$ color cuts listed in Eqs. (6)-(8).

\section{Red optical spectra from the SDSS}

The SDSS imaging data are used to select in a uniform way different classes of objects whose spectra will be taken with the SDSS $2.5 \mathrm{~m}$ telescope (York et al. 2000). The target selection algorithms for spectroscopic follow up are described by Stoughton et al. (2002). The DR7.1 main spectroscopic data base includes data for around 1.2 million objects, and covers $7470 \mathrm{deg}^{2}$. The wavelength coverage is from 3800 to $9200 \AA$ with resolution $\lambda /(\Delta \lambda)=1800$. The signal-to-noise ratio is better than $4 \mathrm{pixel}^{-1}$ at $g=20.2$ (Adelman-McCarthy et al. 2007). The spectra distributed by the SDSS have been sky subtracted and corrected for telluric absorption. The spectroscopic data are automatically reduced by the SDSS pipeline software.

Our final selection consisted of 275 objects of which 87 were cross- referenced with, and confirmed to be mostly L dwarfs. So 188 of these objects are new discoveries. Of these, 36 are confirmed through SDSS optical spectra. At first we only found 28 objects with SDSS spectra when we searched for the photometrically selected candidates in the SDSS spectroscopic cata$\log$. Then we searched the spectra of SDSS color selected candidates with SDSS spectra which were removed with 2MASS criteria $(6,7,8)$. We found six objects with SDSS spectra, three M9, one L0 and two L1 dwarfs. In Fig. 2, we can see that the 2MASS criteria $(6,7,8)$ are set for $\mathrm{L}$ dwarfs and some late $\mathrm{M}$ early $\mathrm{L}$ dwarfs will be missed, including these six objects. Another two objects were found with SDSS spectra which are faint and therefore missed by the 2MASS survey. Table 1 lists the SDSS names, SDSS $r, i, z, 2$ MASS $J, H, K$ and SDSS spectral types for these 36 spectroscopically confirmed ultracool dwarfs. Note that we also performed a cross-match with UKIDSS DR4 (see Sect. 5) and that 23 objects without SDSS spectroscopy that were measured in UKIDSS are listed in Table 2. All remaining candidates (i.e. without SDSS spectroscopy or UKIDSS DR4 coverage) are given in Table 7.

In addition to the photometric and spectral type analysis we also derived proper motion constraints for our new sample, using the dual epoch coordinates from the SDSS and 2MASS databases and dividing any movement between the epochs by the observational epoch difference. Standard errors on these proper motions are calculated using the major axes of the position error ellipses from SDSS and 2MASS, and are dominated by the 2MASS positional uncertainties. Systematic astrometry errors between 2MASS-SDSS which have a shorter baseline, could lead to significant errors in calculating proper motions with coordinates and epoch differences, and can not be ignored. To correct the systematic offset of 2MASS-SDSS, we measured average proper motion of reference objects around every targets for which proper motion has been measured. Then we subtracted this average proper motion from the measured proper motion of each corresponding target. The number of reference objects ranged from around 100 to a few hundreds for different targets, with reference objects being selected to have low coordinate errors (mostly $<0.1^{\prime \prime},<0.2^{\prime \prime}$ for the candidates with fewer reference objects). Reference objects are within $12^{\prime}, 15^{\prime}$ or $20^{\prime}$ of our targets depends on availability. These proper motions are also given in Tables 1 and 3 and 7. Their quality and accuracy is assessed in Sect. 5 through comparison with additional epoch image data and measurement of motion relative to nearby sources surrounding each ultracool dwarf (see also Cols. 10 and 11 in Table 1).

For the $11 \mathrm{M} 9$ and $25 \mathrm{~L}$ dwarfs with SDSS spectra. We assigned their spectral types by comparison with the SDSS spectral sequence of previously found M, L and T dwarfs as shown in Fig. 3, the dwarf classification scheme of Kirkpatrick et al. (1999), SDSS spectra of M and L dwarfs published by Hawley et al. (2002) and the low-mass dwarf template spectra from SDSS (Bochanski et al. 2007). One of the major points we have considered in the comparison is the shape of the normalized spectrum, such as the width of KI region around $7700 \AA$ and the relative flux in the region from $8400 \AA$ to 


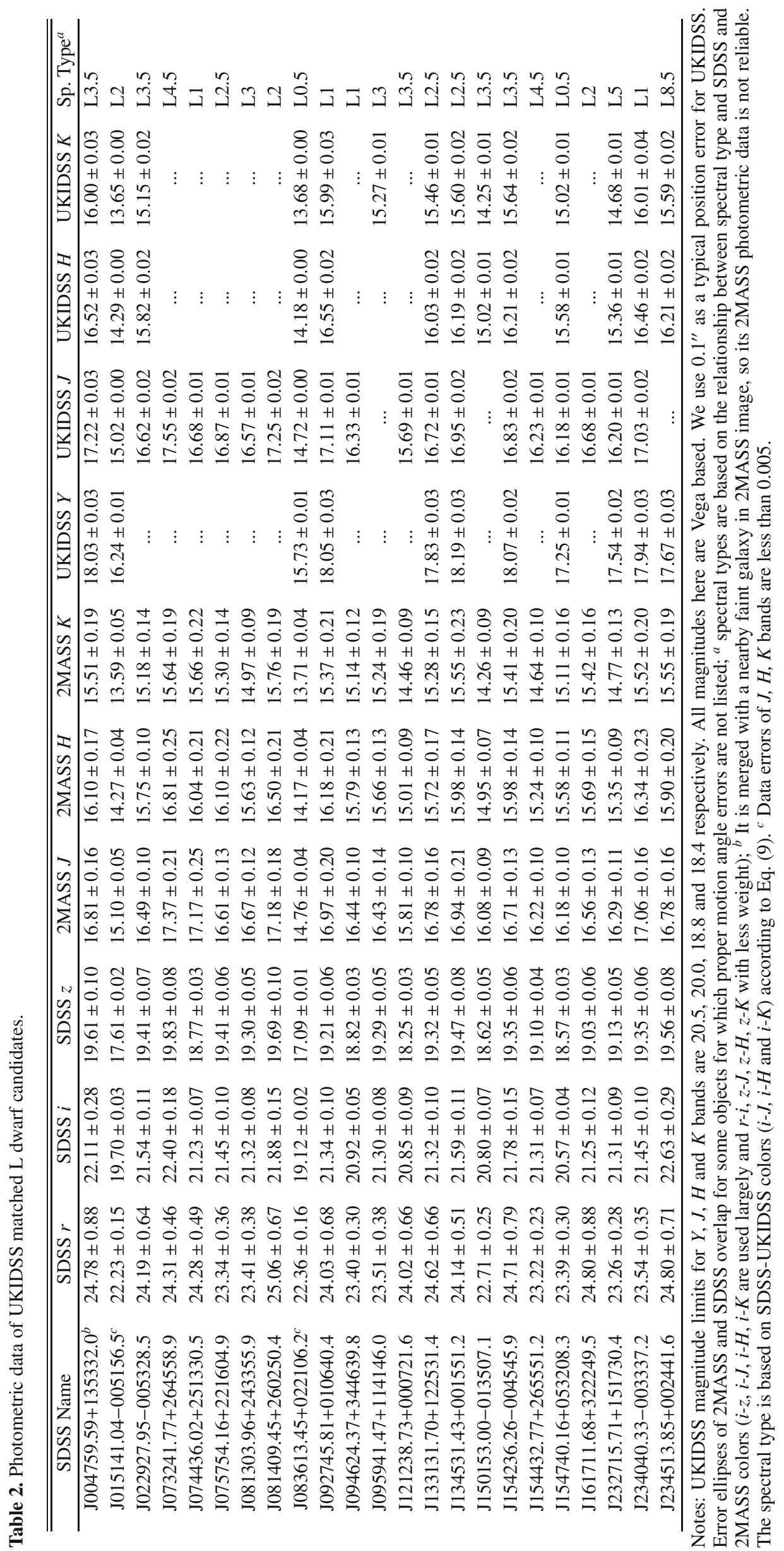


Table 3. Seven objects with SDSS spectra matched in UKIDSS.

\begin{tabular}{ccccccc}
\hline \hline SDSS Name & UKIDSS $Y$ & UKIDSS $J$ & UKIDSS $H$ & UKIDSS $K$ & $\begin{array}{c}\text { Proper motion }^{a} \\
\left(\mathrm{yr}^{-1}\right)\end{array}$ & $\begin{array}{l}\text { Proper motion } \\
\text { Angle }^{b}\end{array}$ \\
\hline SDSS J012052.58+151827.3 & $17.11 \pm 0.02$ & $16.09 \pm 0.01$ & $15.50 \pm 0.01$ & $14.92 \pm 0.01$ & $0.04 \pm 0.02$ & $105 \pm 36$ \\
SDSS J084751.48+013811.0 & $17.38 \pm 0.02$ & $16.09 \pm 0.01$ & $15.22 \pm 0.01$ & $14.45 \pm 0.01$ & $0.03 \pm 0.02$ & $242 \ldots$ \\
SDSS J090347.55+011446.0 & $17.58 \pm 0.02$ & $\ldots$ & $15.59 \pm 0.01$ & $14.96 \pm 0.01$ & $0.03 \pm 0.02$ & $259 \ldots$ \\
SDSS J091714.76+314824.8 & $\ldots$ & $16.36 \pm 0.01$ & $\ldots$ & $\ldots$ & $0.03 \pm 0.03$ & $345 \ldots$ \\
SDSS J100817.07+052312.9 & $\ldots$ & $\ldots$ & $16.36 \pm 0.02$ & $15.89 \pm 0.03$ & $0.21 \pm 0.03$ & $139 \pm 10$ \\
SDSS J154502.87+061807.8 & $17.35 \pm 0.02$ & $16.21 \pm 0.01$ & $15.62 \pm 0.01$ & $15.07 \pm 0.01$ & $0.14 \pm 0.04$ & $251 \pm 25$ \\
SDSS J155215.38+065041.5 & $\ldots$ & $\ldots$ & $15.93 \pm 0.01$ & $15.32 \pm 0.01$ & $0.02 \pm 0.04$ & $356 \ldots$ \\
\hline
\end{tabular}

${ }^{a}$ SDSS-UKIDSS data-base proper motions - found by dividing the difference between the SDSS and UKIDSS coordinates (from the respective databases) by the observational epoch difference. Standard errors are calculated using the major axes of the position error ellipses from SDSS and UKIDSS $;{ }^{b}$ error ellipses of SDSS and UKIDSS overlap for some objects for which position angle errors are not meaningful.

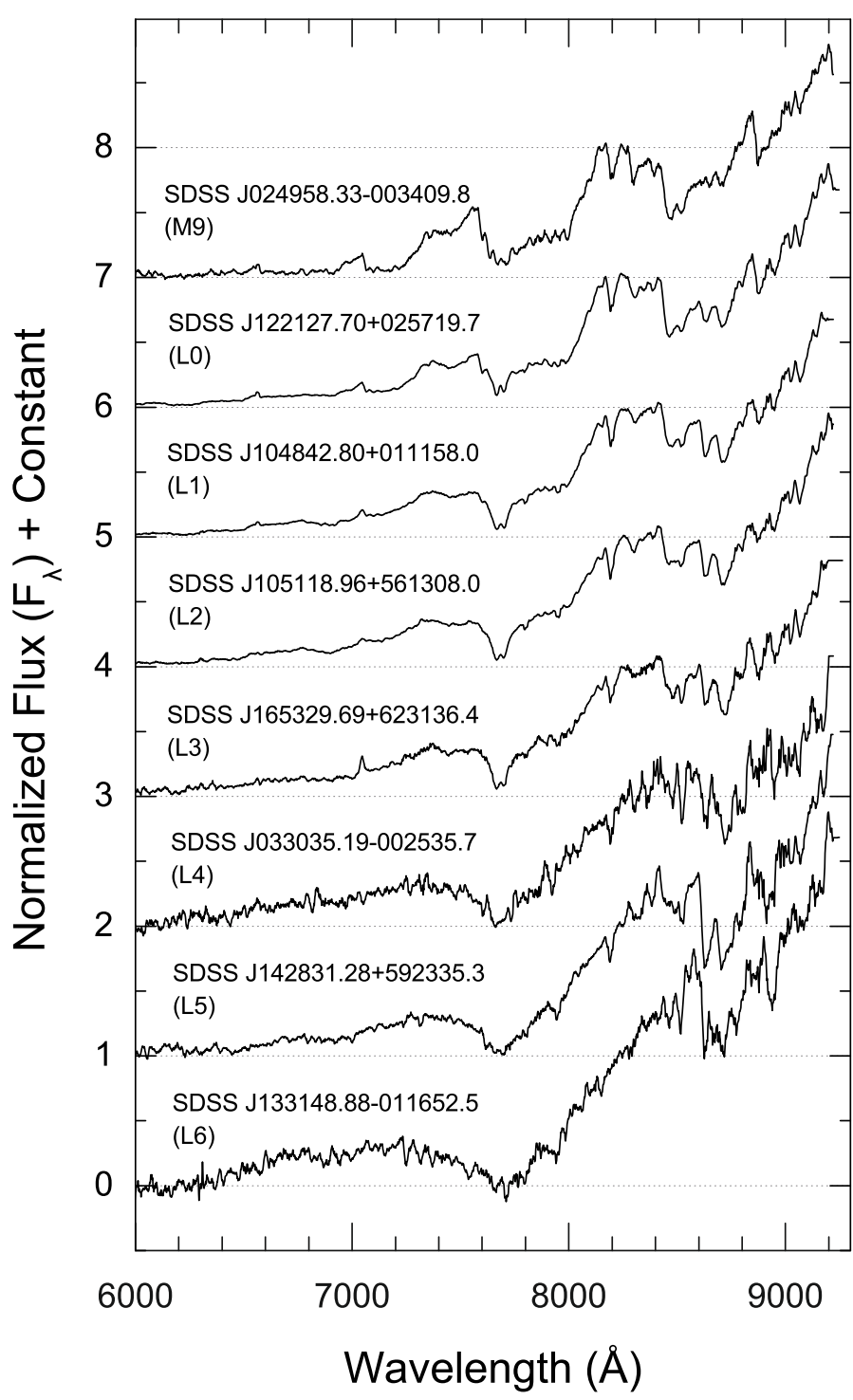

Fig. 3. SDSS spectra of 8 previously found $M$ and $L$ dwarfs, used for spectral typing. All spectra have been normalized to one at $8250 \AA$ and vertically offset for clarity.

$9000 \AA$. Another major point is the slope of the spectrum in the band from $8700 \AA$ to $9200 \AA$. The last criterion is the depth of absorption lines which can be recognized in some spectra, such as NaI $\lambda \lambda 8183,8195, \mathrm{CrH} \lambda 8661, \mathrm{FeH} \lambda 8692$ and CsI $\lambda \lambda 8521,8944$. NaI $\lambda \lambda 8183,8195$ is a major feature of late
$\mathrm{M}$ dwarfs. $\mathrm{CrH} \lambda 8661$ is equal in strength to $\mathrm{FeH} \lambda 8692$ for L4 dwarfs, and stronger for L5 dwarfs. CsI keeps strengthening from L1 to L8 type (Kirkpatrick et al. 1999). Finally, to double check the spectral type of each spectrum, we subtract the spectrum of a ultra-cool dwarf which has the same spectral type and has a good quality, and find a good agreement within our errors.

We note that our selected objects can not be giants because of the presence of the high gravity features such as KI, NaI and $\mathrm{FeH}$, which are characteristic of dwarfs (e.g., Bessell 1991). Figure 3 shows 8 spectra of previously found M, L and T dwarfs as a comparison. SDSS J1428+5923 was recently confirmed and spectral-typed using its infrared spectrum as an L5 dwarf using the Two Micron Proper Motion (2MUPM) survey, under the name 2MASS J14283132+5923354 (Schmidt et al. 2007). SDSS J0249-0034, SDSS J1048+0111, SDSS J1653+6231 and SDSS J1331-0116 were classified by Hawley et al. (2002). SDSS J1221+0257 and SDSS J1051+5613 were discovered by Reid et al. (2008), and SDSS J0330-0025 was discovered by Fan et al. (2000).

Figures 4 and 5 shows the SDSS spectra of the $36 \mathrm{M}$ and $\mathrm{L}$ dwarfs found in this work. The spectra shortward of $6000 \AA$ are flat and noisy, and are not shown. The spectral types of our candidates extend from M9 to L6, and the spectral typing errors are estimated as about $\pm 1 \sim 2$ sub-type. These SDSS spectra have been smoothed by 11 pixels and have been normalized to one at $8250 \AA$. There is a straight line in the spectrum of SDSS J1036+3724 which is an artifact. There is another artificial straight line in the spectrum of SDSS J1431+1436 across $8400 \AA$ A. The spectra of SDSS J0903+0114, SDSS J1329+5317, SDSS J1410+1329 and SDSS J1720+6155 are noisy which made it more difficult to assign their spectral types.

Using the relationship between absolute $J$ and $z$ band magnitudes and spectral types (Hawley et al. 2002), we estimated the approximate distance of the 36 new M and L dwarfs. Generally, early and mid L dwarfs in our sample are between 25 and $100 \mathrm{pc}$, and the $\mathrm{M}$ dwarfs beyond $100 \mathrm{pc}$.

\section{Color-spectral type relationships}

To estimate the spectral types of our ultra-cool dwarf candidates without spectra, we need to know the relationships between spectral types and colors. Hawley et al. (2002) gave the correlation between spectral type and average color for each subtype range from M0 to T6. The relationship is good for M dwarfs, but has large errors for $\mathrm{L}$ and $\mathrm{T}$ dwarfs. With a much larger number of $\mathrm{L}$ and $\mathrm{T}$ dwarfs now available, we made a study of the relationships between spectral types of $\mathrm{L}$ and $\mathrm{T}$ dwarfs and their colors from SDSS and 2MASS. To construct these relationships 

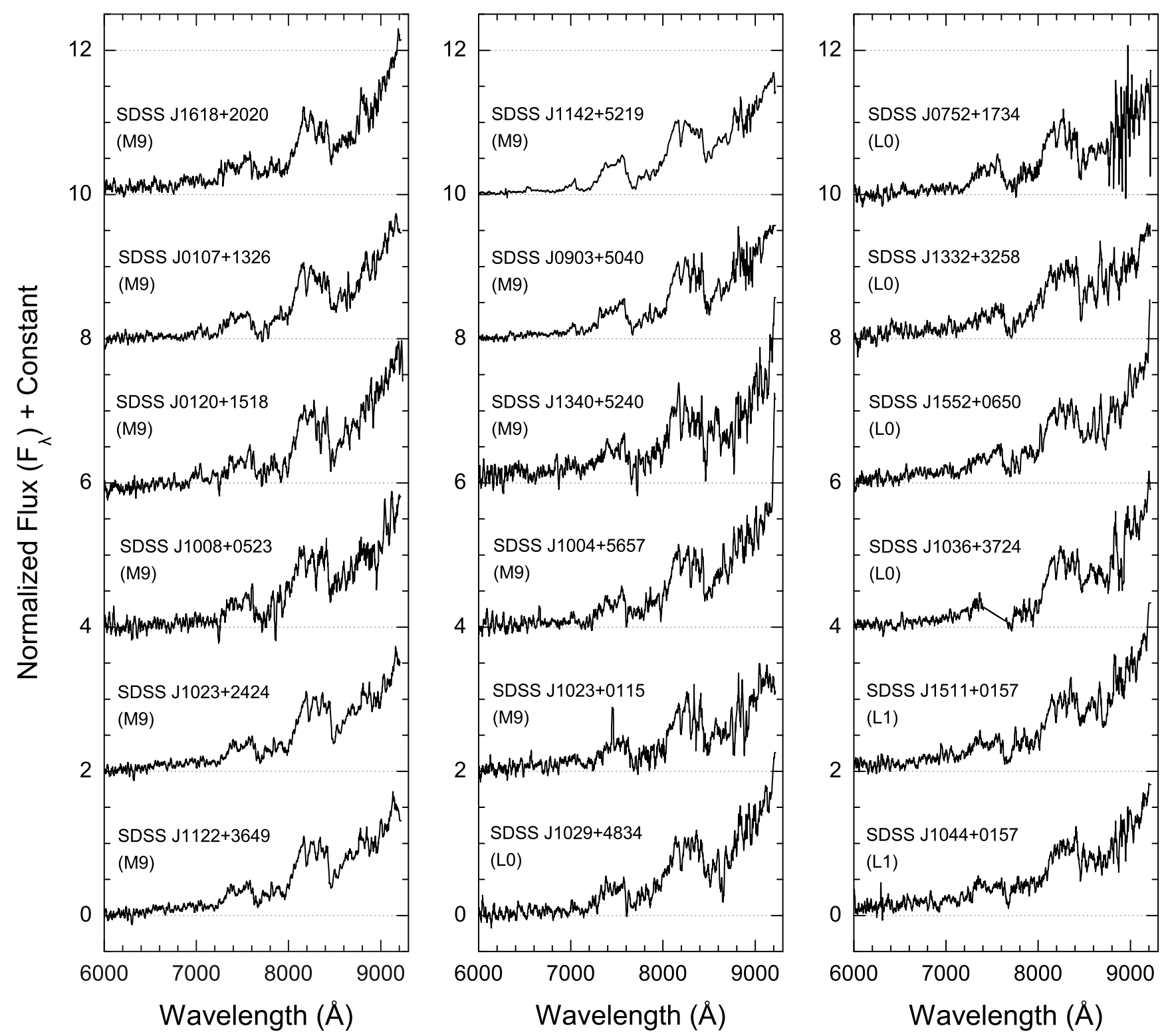

Fig. 4. The SDSS spectra of new M and L dwarfs. Spectral types are given in brackets. The spectra are normalized to one at $8250 \AA$ and offset from one another for clarity. All the spectra are taken from the SDSS archive.

we used the same data set as that for the photometric selection criteria (see section 2). SDSS colors $r-i, i-z, 2$ MASS colors $J-H$, $H-K$ and optical-near infrared colors $i-J, i-H, i-K, z-J, z-H, z-K$ are involved. We fit these relationships with a united polynomial equation,

color $=a+b($ type $)+c(\text { type })^{2}$

where type is a number designed to incompass the full range of M, L and T spectral classes (type $=10$ for L0, 15 for L5, 20 for T0, 25 for T5). Polynomial parameters $a, b$ and $c$ are different for different color ranges and our range of calculated values can be found in Table 4. As well as spectral type ranges, correlation coefficient $R$ and sensitivity indices for the fitting equations are also available in Table 4, where the sensitivity index is defined as the rate of change of color with spectral type, and is thus an indication of the usefulness of a color as a spectral type estimator. Calculated values of the various colors are presented in Table 5 for spectral sub-types between L0 and T7.5. The $i-z, i-J, i-H$ and $i-K$ colors are the most sensitive to spectral type, and the first three of these are plotted as a function of spectral type in Fig. 6.

\section{Cross matching the new sample with UKIDSS}

To provide an additional epoch of deeper near infrared measurements that could improve candidate characterization (particularly for candidates without SDSS spectroscopy), we crossmatched our SDSS DR7 candidates with the Fourth Data Release of the UKIDSS Large Area Survey (Lawrence et al. 2007). UKIDSS magnitude limits for the $Y, J, H$ and $K$ bands are $20.5,20.0,18.8$ and 18.4 respectively. We found that 23 of our candidates (without SDSS spectra) and 7 of our spectroscopically confirmed objects have UKIDSS DR4 counterparts. Table 2 lists the SDSS names, $r, i, z$, 2MASS $J, H, K$, UKIDSS $Y, J, H, K$ and color-estimated spectral types (see Sect. 4) for the objects without spectroscopy, and Table 3 presents the additional UKIDSS information for 7 of the spectroscopic objects from Table 1.

Figure 7 shows the $Y-J$ versus $J-H$ diagram for the 15 candidates that had UKIDSS $Y$ detections. In general these all had UKIDSS YJH magnitudes but in 2 cases we had to transform a 2MASS $J$ into a UKIDSS $J$ following the 

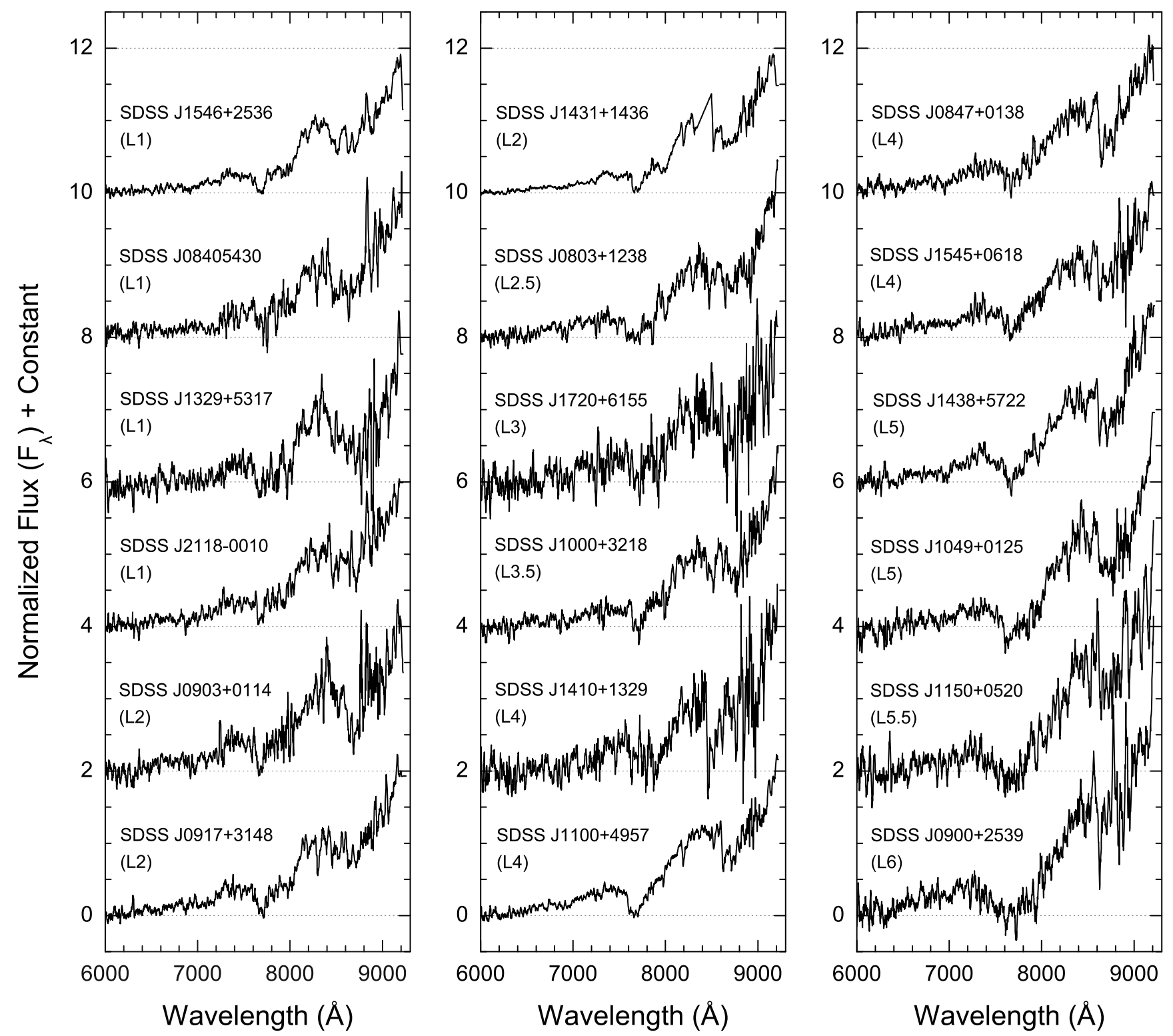

Fig. 5. The SDSS spectra of new M and L dwarfs. Same as Fig. 4.

Table 4. Parameters of fitting equations for color-type relationships.

\begin{tabular}{crrrccc}
\hline \hline Color & $a$ & $b$ & $c$ & $\begin{array}{c}\text { type } \\
\text { Range }\end{array}$ & $R$ & $\begin{array}{c}\text { Sensitivity } \\
\text { Index }^{a}\end{array}$ \\
\hline$r-i$ & 2.77242 & 0.01930 & -0.00355 & {$[10,27.5]$} & 0.62 & 0.073 \\
$i-z$ & 0.89637 & 0.00812 & 0.00582 & {$[13,25]$} & 0.89 & 0.225 \\
$i-J$ & 4.46097 & -0.12872 & 0.01099 & {$[10,25]$} & 0.94 & 0.253 \\
$i-H$ & 2.68743 & 0.22692 & 0 & {$[10,25]$} & 0.92 & 0.227 \\
$i-K$ & 2.04847 & 0.39856 & -0.00567 & {$[10,25]$} & 0.90 & 0.200 \\
$z-J$ & 1.32530 & 0.10725 & 0 & {$[10,13.5]$} & 0.59 & 0.107 \\
$z-J$ & 0.61620 & 0.10826 & 0 & {$[19,26]$} & 0.81 & 0.107 \\
$z-H$ & 4.23705 & -0.31004 & 0.02038 & {$[10,14]$} & 0.70 & 0.158 \\
$z-H$ & 4.60067 & -0.03962 & 0 & {$[17.5,27.5]$} & 0.53 & 0.040 \\
$z-K$ & 3.93079 & -0.21325 & 0.01860 & {$[10,14]$} & 0.72 & 0.235 \\
$z-K$ & 6.96601 & -0.13083 & 0 & {$[17.5,27.5]$} & 0.72 & 0.080 \\
$H-K$ & 0.15050 & 0.03638 & 0 & {$[10,15]$} & 0.40 & 0.040 \\
$H-K$ & 2.19557 & -0.08809 & 0 & {$[17,25]$} & 0.66 & 0.088 \\
$J-H$ & 0.06224 & 0.06903 & 0 & {$[10,14]$} & 0.49 & 0.088 \\
$J-H$ & 5.58544 & -0.21707 & 0 & {$[20,25]$} & 0.90 & 0.100 \\
\hline
\end{tabular}

Notes: the united polynomial fitting equation of color-spectral type relationships is: color $=a+b($ type $)+c(\text { type })^{2}$, type $=10$ for L0, 15 for L5, 20 for T0, 25 for T5; ${ }^{a}$ Sensitivity indices are the rates of change of color ranges with spectral ranges covered by fitting lines. 

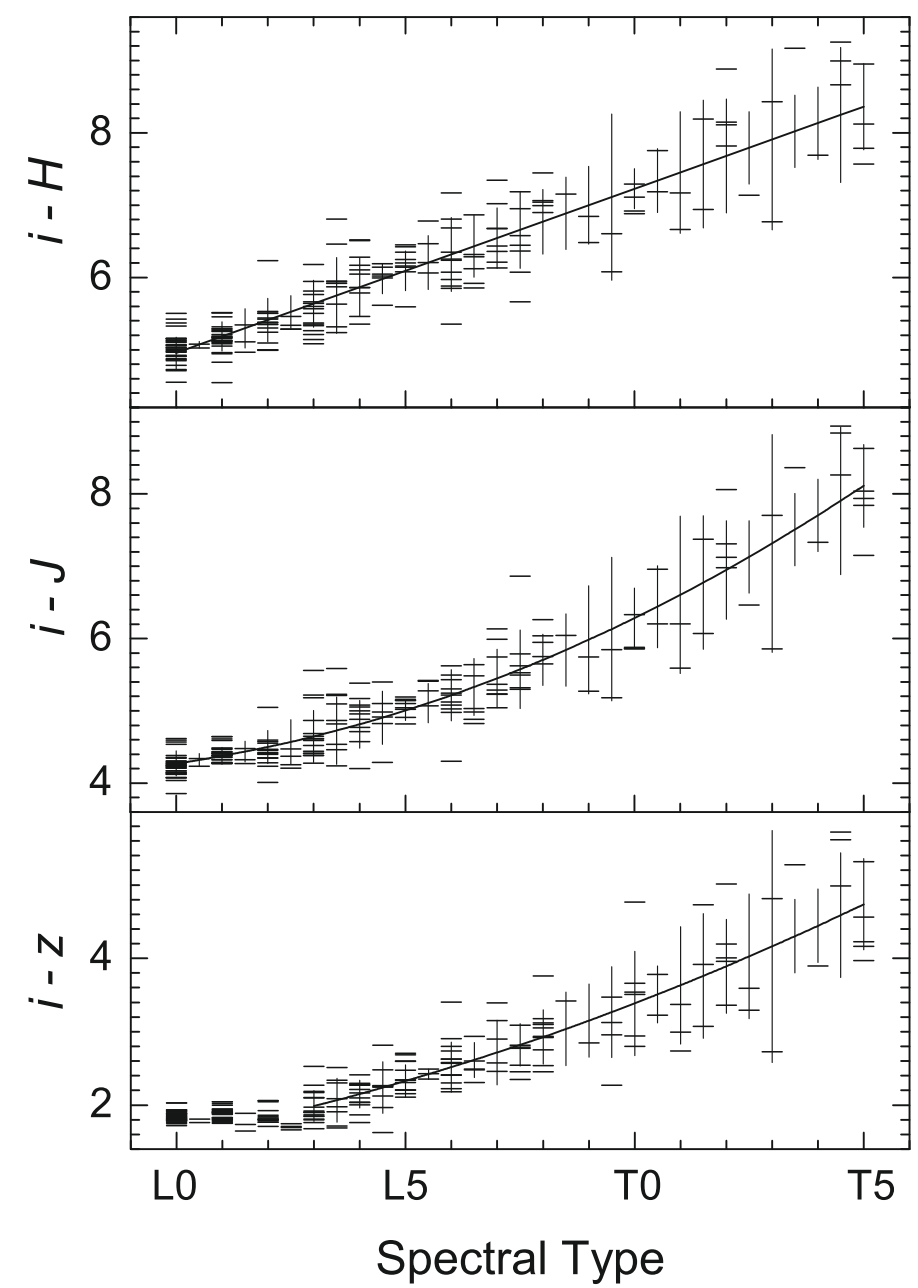

Fig. 6. Polynomial fitting for color-spectral type relationships are indicated with a solid line. The error bars of fitted colors indicate the standard deviation at each subtype. We used 0.5 as the error, if there is only one object available for a given subtype.

conversion of Hewett et al. (2006). This 2-color diagram can provide additional information on ultra-cool dwarf spectral types (e.g. Hewett et al. 2006). For example, the SDSS/2MASS colors of SDSS J2345-0024 suggest a spectral type between L7 and T2. However, Figure 7 suggests that SDSS J2345-0024 is more likely an early $\mathrm{T}$ dwarf than a late L dwarf.

To further assess the proper motions that we derive for all candidates using the coordinate and epoch information stored in the SDSS and 2MASS databases, we have measured additional proper motion constraints using several combinations of multi-epoch data (SDSS/UKIDSS, 2MASS/UKIDSS and 2MASS/SDSS combinations), as well as by measuring the motion of our ultracool dwarf candidates with respect to reference objects within $6^{\prime}$ in the SDSS $z$ and UKIDSS $Y, J, H$ and $K$ images. For the relative proper motions, we used the Iraf routines GEOMAP and GEOXYTRAN to transform the pixel coordinates from the SDSS images into the pixel coordinate system of the UKIDSS images. Visual inspection of the image data revealed a small number of problematic sources. SDSS J0047+1353 is merged with a very nearby galaxy in the 2MASS image. In the main however, four separate proper motion measurements were made (combining $z / Y, z / J, z / H$ and $z / K$ ) where possible, and an average proper motion taken. UKIDSS centroiding accuracy was estimated from the standard deviation of these four measurements, and we also factored in a centroiding uncertainty associated with the SDSS $z$-band epoch, which we estimated to be $0.1^{\prime \prime}$ (1/4 of an SDSS pixel) for these faint sources. The relative proper motions measured from 2MASS and SDSS images are given in Cols. 10 and 11 of Table 1 (for the spectroscopically confirmed objects). The relative proper motions measured from UKIDSS and SDSS images are given in Cols. 2 and 3 of Table 6. Database proper motions (i.e. calculated from cross-database coordinate/epoch information) for UKIDSS/SDSS, 2MASS/UKIDSS, and 2MASS/SDSS database combinations are given in Cols. 4-9 of Table 6, and for the UKIDSS/SDSS combination in Cols. 6, 7 of Table 3. A correction for systematic coordinate uncertainties between UKIDSS and 2MASS/SDSS was decreased as before (see Sect. 3). Figure 8 shows average proper motions of reference objects of objects in Table 6 which proper motions measured with 2MASS-SDSS-UKIDSS databases. The proper motion offsets of 2MASS-UKIDSS with the longest baseline have the smallest offsets $\left(<0.008^{\prime \prime} \mathrm{yr}^{-1}\right)$ which indicated proper motion with a baseline of longer than 5 years (e.g. 2MASS-UKIDSS) will be a very good way for identified ultracool dwarfs.

- In most cases the 2MASS-SDSS proper motions calculated from the databases are in reasonable agreement with our derived relative proper motions to within the uncertainties. However, the uncertainties associated with the relative proper motions are often larger. This is because in general there is a reduced number of useful reference sources in the 2MASS images. This can sometimes be compounded if a sources is close to the edge of a 2MASS strip, since the number of reference sources can be reduced still further. We thus conclude that the database proper motions are to be preferred when combining 2MASS and SDSS survey data.

- In general, the UKIDSS/SDSS, 2MASS/UKIDSS and 2MASS/SDSS database proper motions agree well to within their uncertainties, except in a limited number of cases, where we find that on closer inspection the baseline between the database epochs is low and the 2MASS sources themselves are near the 2MASS detection limit. The 2MASS/SDSS database proper motions thus stand up reasonably well when compared to those measured from a combination of, on average, higher signal-to-noise imaging data.

- The UKIDSS-SDSS proper motions calculated from the databases are in very good agreement with our derived relative proper motions to within the uncertainties, and the estimated uncertainties from each method are comparable. This result is not surprising, but provides a useful verification for our database proper motion calculations.

We thus conclude that overall, our 2MASS-SDSS proper motions calculated from the databases offer a good balance of reasonably accurate measurements over a relatively large sky area.

\section{Discussion}

Although our photometric selection criteria have been shown to be optimized for mid-late L dwarfs (see Fig. 1), most of the sample that had SDSS spectra are actually late M and early L dwarfs. This partly results from a luminosity bias since later, less luminous L dwarfs are only detected by SDSS in a smaller volume. SDSS targeting priority for these objects was primarily determined through brightness, so our spectroscopic sample is reasonably close to a magnitude limited subset of our full photometric selection. However, in addition, later $\mathrm{L}$ dwarfs are redder, 


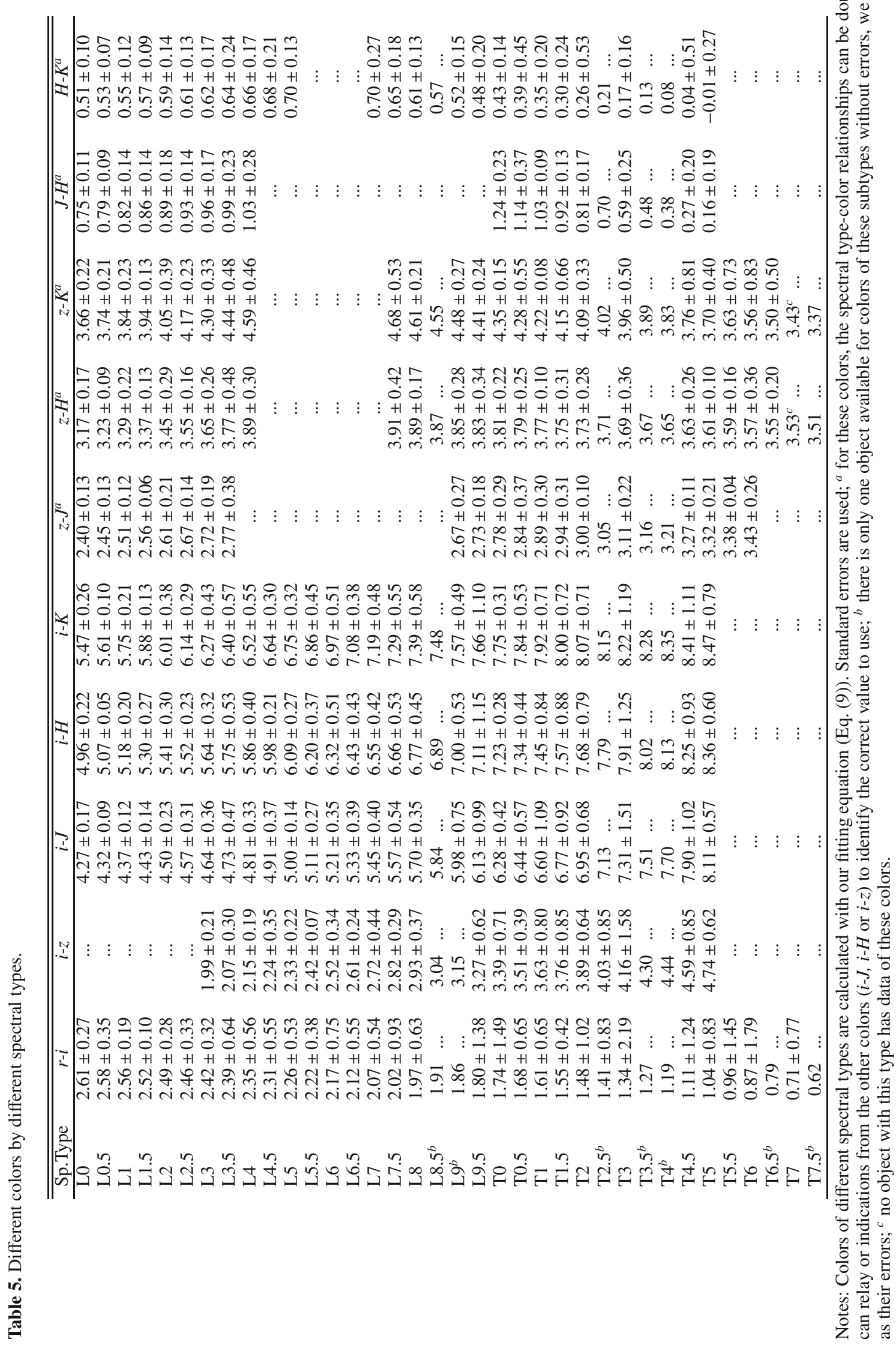




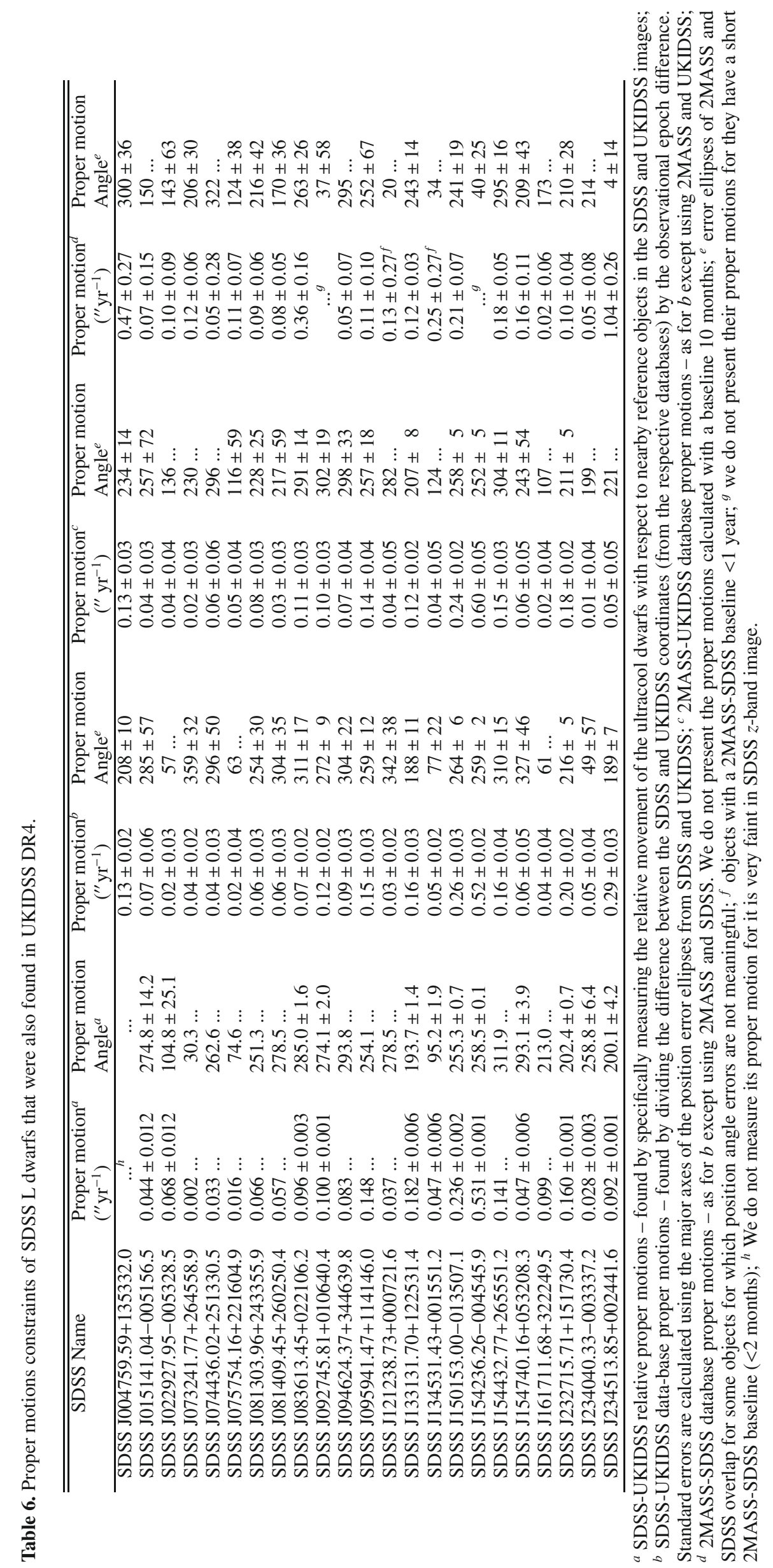


Table 7. SDSS and 2MASS photometry of 129 ultra-cool dwarf candidates.

\begin{tabular}{|c|c|c|c|c|c|c|c|c|c|}
\hline SDSS Name & SDSS $r$ & SDSS $i$ & $\operatorname{SDSS} z$ & 2MASS $J$ & 2MASS $H$ & 2MASS $K$ & $\begin{array}{l}\text { Proper motion } \\
\quad\left({ }^{\prime \prime} \mathrm{yr}^{-1}\right)\end{array}$ & $\begin{array}{l}\text { Proper motion } \\
\text { Angle }\end{array}$ & $\begin{array}{l}\text { Sp. Type } \\
\text { by Colors }\end{array}$ \\
\hline SDSS J073813.07+155304.7 & $23.76 \pm 0.42$ & $21.26 \pm 0.08$ & $19.25 \pm 0.06$ & $16.89 \pm 0.20$ & $16.07 \pm 0.21$ & $15.37 \pm 0.17$ & $0.04 \pm 0.04$ & $184 \ldots$ & $\mathrm{L} 1$ \\
\hline & $23.60 \pm 0.56$ & 0.15 & $39 \pm 0.07$ & $17.31 \pm 0.18$ & $5.67 \pm 0.21$ & $.95 \pm 0.23$ & & 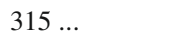 & L0 \\
\hline SDSS J074838.61+174332.9 & $23.89 \pm 0.46$ & $21.35 \pm 0.07$ & $19.06 \pm 0.05$ & $16.27 \pm 0.11$ & $15.18 \pm 0.09$ & $14.42 \pm 0.09$ & $.07 \pm 0.02$ & $264 \pm 14$ & L7 \\
\hline SDSS J075635.25+363033.6 & $22.26 \pm 0.20$ & $19.85 \pm 0.06$ & $17.07 \pm 0.01$ & $15.24 \pm 0.04$ & $14.61 \pm 0.05$ & $14.09 \pm 0.05$ & $0.11 \pm 0.11$ & $149 \ldots$ & L2.5 \\
\hline SDSS J075752.70+091410.0 & $22.86 \pm 0.27$ & $20.62 \pm 0.05$ & $18.57 \pm 0.03$ & $15.86 \pm 0.08$ & $14.83 \pm 0.07$ & $14.09 \pm 0.06$ & $0.14 \pm 0.02$ & $249 \pm 8$ & L4 \\
\hline SDSS J075923.05+462007.4 & $24.18 \pm 0.66$ & $21.44 \pm 0.12$ & $19.30 \pm 0.06$ & $17.00 \pm 0.16$ & $16.09 \pm 0.13$ & $15.86 \pm 0.23$ & $0.23 \pm 0$ & $297 \ldots$ & L2 \\
\hline SDSS J080020.39+360627.1 & $23.61 \pm 0.43$ & $21.24 \pm 0.08$ & $19.23 \pm 0.06$ & $16.64 \pm 0.13$ & $15.93 \pm 0.16$ & $15.62 \pm 0.19$ & & $141 \pm 42$ & L2.5 \\
\hline SDSS J080138.61+372205.8 & $23.00 \pm 0.23$ & $20.59 \pm 0.08$ & $18.21 \pm 0.02$ & $16.83 \pm 0.22$ & $15.84 \pm 0.22$ & $15.45 \pm 0.19$ & $0.22 \pm 0.13$ & $254 \pm 35$ & $\ldots$ \\
\hline SDSS J080252.73+051058.3 & $23.59 \pm 0.64$ & $20.72 \pm 0.09$ & $18.67 \pm 0.06$ & $16.16 \pm 0.11$ & $15.30 \pm 0.09$ & $14.91 \pm 0.14$ & $0.06 \pm 0.04$ & $270 \pm 44$ & L2 \\
\hline SDSS J081215.88+504758.3 & $23.15 \pm 0.32$ & $20.71 \pm 0.05$ & $18.69 \pm 0.04$ & $16.37 \pm 0.13$ & $15.70 \pm 0.18$ & $15.28 \pm 0.17$ & $0.24 \pm c$ & $232 \pm 12$ & L0.5 \\
\hline SDSS J081409.11+281909.6 & $24.25 \pm 0.74$ & $21.69 \pm 0.18$ & $19.58 \pm 0.10$ & $17.45 \pm 0.23$ & $16.47 \pm 0.21$ & $15.79 \pm 0.18$ & .07 & & L0.5 \\
\hline SDSS J081825.78+374103.1 & $23.32 \pm 0.27$ & $21.70 \pm 0.10$ & $19.43 \pm 0.06$ & $16.94 \pm 0.20$ & $16.01 \pm 0.19$ & \pm 0.18 & & & L3.5 \\
\hline SDSS J081843.64+175645.7 & $23.14 \pm 0.34$ & $21.61 \pm 0.15$ & $19.44 \pm 0.09$ & $17.01 \pm 0.19$ & $16.02 \pm 0.19$ & $15.45 \pm 0.17$ & $0.07 \pm 0.05$ & $349 \pm 47$ & L2.5 \\
\hline SDSS J081905.47+493118.5 & $23.75 \pm 0.54$ & $21.23 \pm 0.09$ & $19.19 \pm 0.06$ & $17.09 \pm 0.23$ & $16.51 \pm 0.31$ & $15.56 \pm 0.21$ & $\ldots$ & 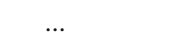 & L0 \\
\hline SDSS J081951.43+543155.1 & $23.71 \pm 0.50$ & $21.02 \pm 0.08$ & $18.97 \pm 0.06$ & $16.97 \pm 0.19$ & $15.91 \pm 0.14$ & $15.45 \pm 0.20$ & $0.13 \pm 0.06$ & $179 \pm 30$ & L1 \\
\hline $.29+280648.0$ & $23.22 \pm 0.36$ & $21.22 \pm 0.09$ & $19.12 \pm 0.06$ & 0.21 & $=0.19$ & 15.4 & $8 \pm 0.10$ & $240 \ldots$ & $\mathrm{L}$ \\
\hline SDSS J082213.75+510120.2 & $23.46 \pm 0.43$ & $20.85 \pm 0.07$ & $18.81 \pm 0.05$ & $16.57 \pm 0.12$ & $15.66 \pm 0.12$ & $15.17 \pm 0.12$ & $\ldots$ & $\ldots$ & L0.5 \\
\hline SDSS J083301.44+445107.6 & $23.45 \pm 0.43$ & $21.19 \pm 0.08$ & $19.12 \pm 0.05$ & $16.54 \pm 0.14$ & $15.96 \pm 0.19$ & $15.42 \pm 0.20$ & $0.56 \pm 0.22$ & $152 \pm 23$ & L2 \\
\hline SDSS J084537.80+293137.2 & $23.74 \pm 0.42$ & $21.47 \pm 0.11$ & $19.42 \pm 0.06$ & $16.74 \pm 0.12$ & $15.97 \pm 0.13$ & $4 \pm 0.12$ & $0.07 \pm 0.04$ & $124=$ & L2.5 \\
\hline SDSS J084737.45+462443.8 & $23.16 \pm 0.50$ & $.92 \pm 0.09$ & $7 \pm 0.07$ & 0.17 & 0.19 & 0.23 & & & \\
\hline SDSS J0847 & $23.08 \pm 0.44$ & $20.83 \pm 0.08$ & $18.79 \pm 0.04$ & 0.14 & 0.15 & 15.2 & & & L0 \\
\hline SDSS J084838.93+484025.4 & $23.07 \pm 0.27$ & $21.04 \pm 0.07$ & $19.03 \pm 0.05$ & $16.59 \pm 0.13$ & $15.77 \pm 0.13$ & $15.29 \pm 0.15$ & & & L2 \\
\hline SDSS J085048.96+173210.9 & $23.33 \pm 0.30$ & $20.89 \pm 0.06$ & $18.88 \pm 0.05$ & $16.57 \pm 0.12$ & $15.93 \pm 0.15$ & $15.23 \pm 0.11$ & $0.15 \pm 0.03$ & $354 \pm 12$ & L0.5 \\
\hline SDSS J085117.58+583226.6 & $24.25 \pm 0.76$ & $21.59 \pm 0.13$ & $19.53 \pm 0.09$ & 17.0 & \pm 0.23 & 15.8 & .07 & & L1.5 \\
\hline SDSS J0851 & $24.36 \pm 0.65$ & $21.79 \pm 0.11$ & $19.58 \pm 0.05$ & $16.7 \mathrm{c}$ & $=0.15$ & 15.0 & & 309 & L5 \\
\hline SDSS J085711.43+415928.6 & $23.72 \pm 0.53$ & $21.46 \pm 0.11$ & $19.39 \pm 0.07$ & 17.37 & $16.18 \pm 0.20$ & 15.7 & & & L2 \\
\hline SDSS J090308.17+165935.5 & $24.87 \pm 0.59$ & $21.61 \pm 0.10$ & $19.38 \pm 0.07$ & $16.49 \pm 0.11$ & \pm 0.16 & $15.49 \pm 0.19$ & $0.11 \pm c$ & $129 \pm 20$ & L6 \\
\hline SDSS J090435.90+322918.7 & $23.71 \pm 0.52$ & 0.07 & $19.02 \pm 0.05$ & 16.97 & 0.20 & 0.20 & $0.38 \pm 0.06$ & $294 \pm 10$ & \\
\hline SDSS J090546.54+562311.9 & $23.01 \pm 0.36$ & $0.28 \pm 0.05$ & $18.24 \pm 0.03$ & 0.05 & \pm 0.04 & 0.04 & & 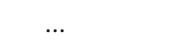 & L4 \\
\hline SDSS J091428.64+230541.2 & $24.66 \pm 0.72$ & $21.26 \pm 0.08$ & $19.14 \pm 0.05$ & $=0.10$ & $15.53 \pm 0.08$ & 0.08 & $0.05 \pm 0.02$ & $311 \pm 22$ & L3 \\
\hline SDSS J091811.89+390216.7 & $23.77 \pm 0.62$ & $22.07 \pm 0.21$ & $19.65 \pm 0.10$ & $17.02=$ & $16.15 \pm 0.21$ & 15.5 & $0.07 \pm$ & $234 \ldots$ & L5.5 \\
\hline SDSS J091816.02+481300.4 & $23.02 \pm 0.23$ & $20.75 \pm 0.05$ & $18.74 \pm 0.05$ & $16.43 \pm 0.12$ & $15.52 \pm 0.12$ & $15.30 \pm 0.14$ & $0.14 \pm 0.06$ & $71 \pm 27$ & L0.5 \\
\hline SDSS J092752.44+572932.5 & $23.90 \pm 0.51$ & $21.37 \pm 0.09$ & $19.36 \pm 0.07$ & $16.65=$ & $15.46 \pm 0.13$ & 0.11 & $\ldots$ & 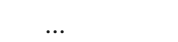 & L3.5 \\
\hline SDSS J092819.74+180 & $23.64 \pm 0.35$ & $22.20 \pm 0.15$ & $19.98 \pm 0.08$ & $17.23=$ & \pm 0.26 & 0.21 & $0.25 \pm 0.05$ & $289 \pm 11$ & L3.5 \\
\hline SDSS J093100.69+605539.6 & $23.64 \pm 0.58$ & $21.10 \pm 0.10$ & $19.00 \pm 0.07$ & $16.48 \pm 0.11$ & $15.69 \pm 0.10$ & 15.3 & & & $\mathrm{~L} 2.5$ \\
\hline SDSS J093204.02+345937.0 & $25.18 \pm 0.73$ & $21.97 \pm 0.22$ & $19.92 \pm 0.10$ & $17.01 \pm 0.18$ & $16.22 \pm 0.19$ & $15.46 \pm 0.21$ & $0.23 \pm 0.07$ & $149 \pm 16$ & L4.5 \\
\hline SDSS J093956.05+242658.7 & $24.85 \pm 0.64$ & $22.07 \pm 0.23$ & $19.69 \pm 0.10$ & $16.97 \pm 0.22$ & $15.93 \pm 0.22$ & $15.53 \pm 0.21$ & $0.36 \pm 0.05$ & $244 \pm 8$ & L6.5 \\
\hline SDSS J094146.38+215843.5 & $25.07 \pm 0.58$ & $21.78 \pm 0.11$ & $19.67 \pm 0.09$ & $16.94 \pm 0.18$ & $15.92 \pm 0.18$ & $=0.17$ & .04 & $244 \pm 26$ & L4 \\
\hline SDSS J094427.33+641037.3 & $22.96 \pm 0.33$ & $20.95 \pm 0.08$ & $18.93 \pm 0.06$ & $16.67 \pm 0.16$ & $15.88 \pm 0.17$ & 15.46 & $0.09 \pm 0.06$ & $296 \pm 38$ & L0 \\
\hline SDSS J094429.58+465254.7 & $22.84 \pm 0.32$ & $21.26 \pm 0.11$ & $19.16 \pm 0.06$ & $17.26 \pm 0.21$ & $16.58 \pm 0.23$ & $16.09 \pm 0.25$ & $0.06 \pm 0.16$ & $322 \ldots$ & $\ldots$ \\
\hline SDSS J095154.19+282040.8 & $23.10 \pm 0.30$ & $21.22 \pm 0.08$ & $19.21 \pm 0.06$ & $16.56 \pm 0.16$ & $16.06 \pm 0.15$ & $15.52 \pm 0.18$ & $0.07 \pm 0.05$ & $298 \pm 44$ & L2 \\
\hline SDSS J095932.74+452330.5 & $23.73 \pm 0.51$ & $21.04 \pm 0.08$ & $19.01 \pm 0.05$ & $15.88 \pm 0.07$ & $14.76 \pm 0.07$ & $=0.04$ & $0.19=$ & 221 & L7.5 \\
\hline SDSS J100132.26+492819.9 & $23.05 \pm 0.25$ & $20.74 \pm 0.05$ & $18.72 \pm 0.04$ & $16.68 \pm 0.13$ & $15.95 \pm 0.14$ & 0.18 & $0.23 \pm 0.09$ & $276 \pm 23$ & $\ldots$ \\
\hline SDSS J100317.30+331922.0 & $24.32 \pm 0.79$ & $21.60 \pm 0.15$ & $19.23 \pm 0.07$ & $16.74 \pm 0.14$ & $15.73 \pm 0.11$ & $15.23 \pm 0.14$ & $0.05 \pm 0.04$ & $75 \pm 56$ & L4 \\
\hline SDSS J100633.74+363919.5 & $23.89 \pm 0.39$ & $21.60 \pm 0.08$ & $19.49 \pm 0.05$ & $17.10 \pm 0.22$ & $15.98 \pm 0.16$ & $15.32 \pm 0.19$ & $0.12 \pm 0.07$ & $184 \pm 32$ & L2.5 \\
\hline SDSS J101134.72+501400.7 & $23.79 \pm 0.49$ & $21.93 \pm 0.15$ & $19.90 \pm 0.10$ & $17.60 \pm 0.29$ & $16.28 \pm 0.20$ & $15.57 \pm 0.17$ & $0.70 \pm 0.22$ & \pm 18 & L2 \\
\hline SDSS J101439.66+252511.3 & $24.45 \pm 0.59$ & $21.14 \pm 0.07$ & $19.10 \pm 0.05$ & $17.24 \pm 0.23$ & $16.19 \pm 0.21$ & $15.83 \pm 0.24$ & $0.09 \pm 0.05$ & $215 \pm 35$ & L0 \\
\hline SDSS J101951.13+044944.1 & $24.10 \pm 0.57$ & $21.80 \pm 0.15$ & $19.48 \pm 0.07$ & $16.85 \pm 0.18$ & $16.18 \pm 0.20$ & $15.54 \pm 0.23$ & $0.57 \pm 0.31$ & $240 \pm 33$ & L4 \\
\hline SDSS J102517.57+285113.6 & $23.64 \pm 0.54$ & $20.95 \pm 0.07$ & $18.92 \pm 0.05$ & $16.54 \pm 0.10$ & $15.83 \pm 0.12$ & $15.31 \pm 0.12$ & $0.11 \pm 0.03$ & $288 \pm 16$ & $\mathrm{~L} 1.5$ \\
\hline SDSS J102546.97+151126.3 & $24.29 \pm 0.70$ & $21.52 \pm 0.15$ & $19.47 \pm 0.07$ & $16.99 \pm 0.15$ & $16.06 \pm 0.17$ & $15.42 \pm 0.15$ & $0.17 \pm 0.04$ & $276 \pm 13$ & L2 \\
\hline SDSS J102935.23+062029.6 & $24.51 \pm 0.55$ & $22.47 \pm 0.19$ & $19.37 \pm 0.05$ & $16.87 \pm 0.22$ & $16.09 \pm 0.21$ & $15.02 \pm 0.16$ & & $212 \pm 75$ & \\
\hline SDSS J102939.69+571544.3 & $24.35 \pm 0.52$ & $21.62 \pm 0.10$ & $19.17 \pm 0.06$ & $16.70 \pm 0.13$ & $15.46 \pm 0.11$ & $14.99 \pm 0.09$ & $0.82 \pm 0.07$ & $95 \pm 5$ & L4.5 \\
\hline SDSS J103908.17+244044.1 & $23.58 \pm 0.34$ & $21.73 \pm 0.11$ & $19.41 \pm 0.06$ & $16.76 \pm 0.14$ & $15.72 \pm 0.13$ & $15.04 \pm 0.11$ & $0.22 \pm 0.03$ & $236 \pm 7$ & L5 \\
\hline SDSS J104808.29+544715.1 & $23.50 \pm 0.40$ & $21.86 \pm 0.14$ & $19.46 \pm 0.07$ & $16.73 \pm 0.19$ & $15.86 \pm 0.20$ & $15.43 \pm 0.19$ & $0.38 \pm 0.11$ & $295 \pm 17$ & L6.5 \\
\hline SDSS J104814.75+135833.3 & $23.78 \pm 0.44$ & $21.94 \pm 0.15$ & $19.43 \pm 0.07$ & $16.90 \pm 0.13$ & $16.01 \pm 0.14$ & $15.34 \pm 0.14$ & & $197 \pm 14$ & L5.5 \\
\hline SDSS J105204.75+172241.2 & $23.14 \pm 0.34$ & $21.27 \pm 0.10$ & $19.21 \pm 0.06$ & $16.71 \pm 0.11$ & $16.04 \pm 0.14$ & $15.53 \pm 0.16$ & $0.31 \pm 0.03$ & $158 \pm 6$ & L1.5 \\
\hline SDSS J105254.02+584951.2 & $23.96 \pm 0.37$ & $21.44 \pm 0.08$ & $19.26 \pm 0.04$ & $16.44 \pm 0.12$ & $15.44 \pm 0.13$ & $14.87 \pm 0.10$ & $0.09 \pm 0.05$ & $54 \pm 37$ & L5 \\
\hline SDSS J110827.31+083801.8 & $23.74 \pm 0.71$ & $22.39 \pm 0.34$ & $19.26 \pm 0.07$ & $16.58 \pm 0.16$ & $15.50 \pm 0.11$ & $15.03 \pm 0.16$ & $0.34 \pm 0.08$ & $217 \pm 14$ & L8.5 \\
\hline
\end{tabular}


Table 7. continued.

\begin{tabular}{|c|c|c|c|c|c|c|c|c|c|}
\hline SDSS Name & $\operatorname{SDSS} r$ & SDSS $i$ & $\operatorname{SDSS} z$ & 2MASS $J$ & 2MASS $H$ & 2MASS $K$ & $\begin{array}{c}\text { Proper motion } \\
\left(1 " \mathrm{yr}^{-1}\right)\end{array}$ & $\begin{array}{l}\text { Proper motion } \\
\text { Angle }\end{array}$ & $\begin{array}{l}\text { Sp. Type } \\
\text { by Colors }\end{array}$ \\
\hline SDSS J111501.36+160701.5 & $22.94 \pm 0.37$ & $20.71 \pm 0.07$ & $18.59 \pm 0.05$ & $16.40 \pm 0.12$ & $15.22 \pm 0.09$ & $14.56 \pm 0.11$ & $0.34 \pm 0.08$ & $248 \pm 14$ & L1 \\
\hline SDSS J111802.89+060703.6 & $24.09 \pm 0.53$ & $21.42 \pm 0.10$ & $19.35 \pm 0.05$ & $17.00 \pm 0.18$ & $16.27 \pm 0.21$ & $15.66 \pm 0.26$ & $0.28 \pm 0.11$ & $133 \pm 23$ & L1.5 \\
\hline SDSS J111910.46+055248.4 & $23.39 \pm 0.33$ & $21.67 \pm 0.11$ & $19.60 \pm 0.06$ & $16.76 \pm 0.16$ & $15.48 \pm 0.11$ & $15.03 \pm 0.15$ & $0.07 \pm 0.16$ & $109 \ldots$ & L5 \\
\hline SDSS J112012.95+212520.4 & $23.50 \pm 0.42$ & $21.65 \pm 0.11$ & $19.53 \pm 0.06$ & $16.90 \pm 0.17$ & $15.79 \pm 0.14$ & $15.34 \pm 0.14$ & $0.13 \pm 0.04$ & $198 \pm 16$ & L4 \\
\hline SDSS J112722.94-003714.4 & $23.53 \pm 0.33$ & $21.34 \pm 0.08$ & $19.24 \pm 0.05$ & $16.81 \pm 0.18$ & $16.12 \pm 0.26$ & $15.35 \pm 0.20$ & $\ldots$ & $\ldots$ & L1.5 \\
\hline SDSS J113022.46+122751.6 & $23.49 \pm 0.48$ & $21.69 \pm 0.13$ & $19.64 \pm 0.10$ & $17.05 \pm 0.18$ & $16.47 \pm 0.22$ & $15.98 \pm 0.24$ & $0.16 \pm 0.11$ & $358 \pm 41$ & L2 \\
\hline SDSS J113639.67+485240.3 & $23.54 \pm 0.31$ & $20.91 \pm 0.05$ & $18.86 \pm 0.03$ & $16.16 \pm 0.10$ & $15.27 \pm 0.11$ & $14.58 \pm 0.08$ & $0.20 \pm 0.06$ & $307 \pm 17$ & L3.5 \\
\hline SDSS J114103.28+632805.9 & $23.57 \pm 0.41$ & $21.50 \pm 0.12$ & $19.46 \pm 0.07$ & $16.72 \pm 0.13$ & $15.94 \pm 0.13$ & $15.62 \pm 0.23$ & $0.45 \pm 0.18$ & $308 \pm 23$ & L3 \\
\hline SDSS J114302.72+190541.9 & $24.77 \pm 0.62$ & $22.76 \pm 0.26$ & $19.77 \pm 0.08$ & $16.77 \pm 0.18$ & $15.80 \pm 0.15$ & $15.01 \pm 0.14$ & $0.26 \pm 0.03$ & $172 \pm 8$ & L9 \\
\hline SDSS J114807.23+390106.9 & $24.12 \pm 0.66$ & $21.23 \pm 0.08$ & $19.19 \pm 0.05$ & $16.92 \pm 0.18$ & $15.96 \pm 0.17$ & $15.52 \pm 0.17$ & $0.20 \pm 0.06$ & $323 \pm 18$ & L1.5 \\
\hline SDSS J115017.36+512502.4 & $24.28 \pm 0.60$ & $22.02 \pm 0.18$ & $19.98 \pm 0.11$ & $16.87 \pm 0.20$ & $16.03 \pm 0.18$ & $15.08 \pm 0.12$ & $0.15 \pm 0.12$ & $221 \pm 51$ & L6 \\
\hline SDSS J115058.98+440917.2 & $24.06 \pm 0.58$ & $21.38 \pm 0.09$ & $19.37 \pm 0.06$ & $17.05 \pm 0.19$ & $16.15 \pm 0.23$ & $15.49 \pm 0.15$ & $0.14 \pm 0.06$ & $272 \pm 26$ & L1 \\
\hline SDSS J115722.81+264119.6 & $23.71 \pm 0.47$ & $21.06 \pm 0.07$ & $18.89 \pm 0.04$ & $16.71 \pm 0.12$ & $15.91 \pm 0.15$ & $15.02 \pm 0.11$ & $0.06 \pm 0.03$ & $263 \pm 25$ & L1 \\
\hline SDSS J115820.75+043501.7 & $22.00 \pm 0.20$ & $20.36 \pm 0.07$ & $17.95 \pm 0.04$ & $15.61 \pm 0.06$ & $14.68 \pm 0.06$ & $14.44 \pm 0.06$ & $0.86 \pm 0.86$ & $316 \ldots$ & L3 \\
\hline SDSS J120136.14+135005.9 & $22.76 \pm 0.17$ & $20.66 \pm 0.05$ & $18.63 \pm 0.04$ & $16.88 \pm 0.13$ & $16.14 \pm 0.17$ & $15.74 \pm 0.16$ & $0.03 \pm 0.04$ & $94 \ldots$ & \\
\hline SDSS J120337.00+445333.4 & $23.85 \pm 0.45$ & $21.74 \pm 0.12$ & $19.52 \pm 0.06$ & $17.43 \pm 0.25$ & $16.55 \pm 0.25$ & $15.56 \pm 0.18$ & $0.24 \pm 0.08$ & $281 \pm 20$ & L0.5 \\
\hline SDSS J121846.56+410016.0 & $24.87 \pm 0.71$ & $21.74 \pm 0.13$ & $19.61 \pm 0.07$ & $16.74 \pm 0.16$ & $15.58 \pm 0.13$ & $15.13 \pm 0.15$ & $0.05 \pm 0.05$ & $118 \ldots$ & L5.5 \\
\hline SDSS J122218.47+364348.4 & $22.61 \pm 0.17$ & $20.21 \pm 0.03$ & $18.15 \pm 0.03$ & $15.97 \pm 0.08$ & $15.27 \pm 0.10$ & $14.85 \pm 0.09$ & $0.27 \pm 0.04$ & $92 \pm 9$ & L0 \\
\hline SDSS J122449.44+502154.1 & $23.98 \pm 0.45$ & $21.62 \pm 0.10$ & $19.14 \pm 0.06$ & $16.53 \pm 0.13$ & $15.66 \pm 0.11$ & $14.86 \pm 0.10$ & $0.48 \pm 0.05$ & $204 \pm 6$ & L6 \\
\hline SDSS J123256.67+484417.0 & $22.21 \pm 0.18$ & $20.54 \pm 0.06$ & $18.54 \pm 0.03$ & $16.47 \pm 0.18$ & $15.59 \pm 0.14$ & $15.32 \pm 0.20$ & $0.44 \pm 0.11$ & $254 \pm 15$ & L0 \\
\hline SDSS J124151.85+561541.6 & $23.44 \pm 0.42$ & $20.86 \pm 0.07$ & $18.81 \pm 0.04$ & $16.42 \pm 0.11$ & $15.61 \pm 0.12$ & $14.95 \pm 0.11$ & $0.04 \pm 0.04$ & $314 \pm 77$ & L2 \\
\hline SDSS J124609.07+294200.1 & $24.07 \pm 0.48$ & $21.30 \pm 0.08$ & $19.26 \pm 0.05$ & $16.97 \pm 0.19$ & $16.12 \pm 0.21$ & $15.65 \pm 0.24$ & $0.02 \pm 0.06$ & $331 \ldots$ & L1 \\
\hline SDSS J124655.54+535342.8 & $23.89 \pm 0.47$ & $21.31 \pm 0.08$ & $19.24 \pm 0.05$ & $16.37 \pm 0.11$ & $15.57 \pm 0.12$ & $14.98 \pm 0.12$ & $0.17 \pm 0.09$ & $160 \pm 33$ & L4 \\
\hline SDSS J125002.99+484834.3 & $23.99 \pm 0.47$ & $21.93 \pm 0.16$ & $19.88 \pm 0.09$ & $17.30 \pm 0.26$ & $15.82 \pm 0.14$ & $15.25 \pm 0.14$ & $0.37 \pm 0.15$ & $263 \pm 24$ & L4 \\
\hline SDSS J125137.76+462026.0 & $22.94 \pm 0.21$ & $20.59 \pm 0.05$ & $18.50 \pm 0.03$ & $15.83 \pm 0.08$ & $14.75 \pm 0.07$ & $14.48 \pm 0.10$ & $0.31 \pm 0.02$ & $172 \pm 4$ & L4 \\
\hline SDSS J125410.69+382546.0 & $23.80 \pm 0.46$ & $21.48 \pm 0.11$ & $19.42 \pm 0.06$ & $16.88 \pm 0.15$ & $16.11 \pm 0.18$ & $15.65 \pm 0.16$ & $0.06 \pm 0.04$ & $239 \pm 47$ & $\mathrm{~L} 2.5$ \\
\hline SDSS J125438.50+434657.2 & $23.70 \pm 0.48$ & $22.22 \pm 0.22$ & $19.47 \pm 0.07$ & $16.81 \pm 0.15$ & $15.35 \pm 0.10$ & $14.81 \pm 0.09$ & $0.20 \pm 0.04$ & $257 \pm 11$ & L8.5 \\
\hline SDSS J130449.88+010627.0 & $23.10 \pm 0.31$ & $21.37 \pm 0.10$ & $19.30 \pm 0.06$ & $16.69 \pm 0.17$ & $15.80 \pm 0.17$ & $15.12 \pm 0.17$ & $0.83 \pm 0.24$ & $125 \pm 17$ & L3 \\
\hline SDSS J131142.11+362923.9 & $23.08 \pm 0.23$ & $20.49 \pm 0.04$ & $18.31 \pm 0.03$ & $15.55 \pm 0.05$ & $14.75 \pm 0.06$ & $14.14 \pm 0.05$ & $0.40 \pm 0.02$ & $279 \pm 3$ & L4 \\
\hline SDSS J131218.20+284643.0 & $24.13 \pm 0.57$ & $21.36 \pm 0.09$ & $19.31 \pm 0.06$ & $17.27 \pm 0.26$ & $16.33 \pm 0.32$ & $15.72 \pm 0.21$ & $0.10 \pm 0.09$ & $219 \pm 58$ & L0 \\
\hline SDSS J132535.68+504007.0 & $24.45 \pm 0.54$ & $21.56 \pm 0.08$ & $19.38 \pm 0.05$ & $16.93 \pm 0.23$ & $15.86 \pm 0.23$ & $15.46 \pm 0.23$ & $0.32 \pm 0.15$ & $288 \pm 27$ & L3 \\
\hline SDSS J133316.06+374421.7 & $23.31 \pm 0.29$ & $21.01 \pm 0.06$ & $18.65 \pm 0.03$ & $15.89 \pm 0.06$ & $14.90 \pm 0.05$ & $14.30 \pm 0.05$ & $0.12 \pm 0.04$ & $76 \pm 18$ & L6.5 \\
\hline SDSS J134210.11+414023.8 & $23.65 \pm 0.35$ & $21.63 \pm 0.11$ & $19.56 \pm 0.07$ & $16.86 \pm 0.13$ & $15.87 \pm 0.12$ & $15.47 \pm 0.14$ & $0.20 \pm 0.04$ & $229 \pm 12$ & L4 \\
\hline SDSS J135640.46+140205.3 & $23.83 \pm 0.35$ & $20.96 \pm 0.08$ & $18.36 \pm 0.03$ & $16.62 \pm 0.15$ & $16.10 \pm 0.22$ & $15.28 \pm 0.15$ & $0.03 \pm 0.06$ & $317 \ldots$ & L1 \\
\hline SDSS J140058.03+234923.9 & $23.67 \pm 0.39$ & $21.51 \pm 0.09$ & $19.48 \pm 0.06$ & $16.73 \pm 0.17$ & $16.12 \pm 0.23$ & $15.38 \pm 0.22$ & $0.06 \pm 0.06$ & $261 \ldots$ & L3 \\
\hline SDSS J140318.98+243718.0 & $23.56 \pm 0.33$ & $21.55 \pm 0.09$ & $19.52 \pm 0.07$ & $17.18 \pm 0.23$ & $16.06 \pm 0.22$ & $15.50 \pm 0.22$ & $0.03 \pm 0.06$ & $194 \ldots$ & L1.5 \\
\hline SDSS J141118.49+294850.4 & $23.52 \pm 0.37$ & $21.07 \pm 0.07$ & $18.76 \pm 0.04$ & $16.20 \pm 0.09$ & $15.43 \pm 0.11$ & $15.09 \pm 0.11$ & $0.28 \pm 0.05$ & $164 \pm 10$ & L3.5 \\
\hline SDSS J141405.84+010710.4 & $23.40 \pm 0.38$ & $21.77 \pm 0.15$ & $19.63 \pm 0.09$ & $16.74 \pm 0.20$ & $15.74 \pm 0.19$ & $15.25 \pm 0.20$ & $\ldots$ & $\ldots$ & L5.5 \\
\hline SDSS J142110.77+472834.3 & $23.92 \pm 0.39$ & $20.40 \pm 0.03$ & $18.33 \pm 0.02$ & $16.16 \pm 0.09$ & $15.33 \pm 0.10$ & $14.98 \pm 0.13$ & $0.07 \pm 0.03$ & $240 \pm 22$ & L0 \\
\hline SDSS J142404.53+184641.4 & $24.16 \pm 0.50$ & $21.41 \pm 0.09$ & $19.34 \pm 0.06$ & $17.08 \pm 0.19$ & $16.44 \pm 0.26$ & $15.50 \pm 0.16$ & $0.01 \pm 0.07$ & $175 \ldots$ & L0.5 \\
\hline SDSS J142527.14+300400.4 & $23.11 \pm 0.25$ & $20.78 \pm 0.05$ & $18.72 \pm 0.03$ & $16.39 \pm 0.12$ & $15.54 \pm 0.13$ & $15.03 \pm 0.12$ & $0.02 \pm 0.04$ & $292 \ldots$ & L1.5 \\
\hline SDSS J142612.86+313039.4 & $23.70 \pm 0.42$ & $21.39 \pm 0.09$ & $19.29 \pm 0.05$ & $16.62 \pm 0.16$ & $15.59 \pm 0.13$ & $14.72 \pm 0.09$ & $0.19 \pm 0.04$ & $224 \pm 11$ & $\mathrm{~L} 4$ \\
\hline SDSS J143412.02+271729.9 & $23.98 \pm 0.62$ & $21.41 \pm 0.11$ & $19.29 \pm 0.07$ & $17.38 \pm 0.24$ & $16.33 \pm 0.21$ & $15.70 \pm 0.21$ & $0.08 \pm 0.07$ & $96 \pm 68$ & L0 \\
\hline SDSS J143636.98+465302.7 & $23.75 \pm 0.38$ & $21.92 \pm 0.11$ & $19.92 \pm 0.08$ & $16.99 \pm 0.16$ & $16.13 \pm 0.15$ & $15.81 \pm 0.20$ & $0.28 \pm 0.07$ & $268 \pm 15$ & L4 \\
\hline SDSS J145052.71+462024.8 & $23.87 \pm 0.45$ & $21.68 \pm 0.10$ & $19.67 \pm 0.09$ & $16.77 \pm 0.15$ & $15.93 \pm 0.17$ & $15.26 \pm 0.14$ & $0.14 \pm 0.07$ & $305 \pm 32$ & L4 \\
\hline SDSS J150651.27+553350.7 & $22.93 \pm 0.32$ & $21.10 \pm 0.10$ & $19.10 \pm 0.08$ & $16.69 \pm 0.12$ & $15.80 \pm 0.13$ & $15.30 \pm 0.15$ & $0.08 \pm 0.11$ & $206 \ldots$ & L2 \\
\hline SDSS J151110.91+434036.3 & $23.48 \pm 0.33$ & $21.59 \pm 0.09$ & $19.29 \pm 0.05$ & $16.60 \pm 0.15$ & $15.47 \pm 0.13$ & $14.70 \pm 0.13$ & $0.24 \pm 0.04$ & $152 \pm 9$ & L5 \\
\hline SDSS J152427.98+024210.1 & $22.76 \pm 0.29$ & $21.21 \pm 0.12$ & $19.16 \pm 0.06$ & $16.98 \pm 0.20$ & $16.39 \pm 0.24$ & $15.35 \pm 0.17$ & $\ldots$ & $\ldots$ & L0 \\
\hline SDSS J152802.91+013949.6 & $23.06 \pm 0.33$ & $21.38 \pm 0.11$ & $19.27 \pm 0.12$ & $16.65 \pm 0.14$ & $15.75 \pm 0.15$ & $15.26 \pm 0.17$ & $\ldots$ & $\ldots$ & L3 \\
\hline SDSS J153607.12+203032.6 & $24.01 \pm 0.53$ & $21.81 \pm 0.14$ & $19.73 \pm 0.10$ & $17.26 \pm 0.21$ & $16.17 \pm 0.20$ & $15.77 \pm 0.24$ & $0.03 \pm 0.07$ & $243 \ldots$ & $\mathrm{L} 2.5$ \\
\hline SDSS J153848.19+360337.5 & $24.05 \pm 0.44$ & $21.68 \pm 0.11$ & $19.56 \pm 0.05$ & $16.64 \pm 0.15$ & $15.76 \pm 0.13$ & $15.29 \pm 0.14$ & $0.06 \pm 0.07$ & $286 \ldots$ & L5.5 \\
\hline SDSS J153941.94+531131.0 & $23.53 \pm 0.44$ & $21.44 \pm 0.12$ & $19.40 \pm 0.07$ & $16.86 \pm 0.16$ & $15.95 \pm 0.17$ & $15.19 \pm 0.18$ & $0.17 \pm 0.09$ & $204 \pm 34$ & $\mathrm{~L} 2$ \\
\hline SDSS J154038.76-001257.1 & $23.11 \pm 0.28$ & $21.60 \pm 0.10$ & $19.35 \pm 0.06$ & $16.80 \pm 0.15$ & $15.67 \pm 0.12$ & $15.05 \pm 0.14$ & $\ldots$ & $\ldots$ & L4 \\
\hline SDSS J154455.20+330145.1 & $22.96 \pm 0.23$ & $20.54 \pm 0.05$ & $18.33 \pm 0.02$ & $15.55 \pm 0.06$ & $14.52 \pm 0.06$ & $13.94 \pm 0.05$ & $0.11 \pm 0.03$ & $25 \pm 15$ & L5 \\
\hline SDSS J154623.27+333803.2 & $24.03 \pm 0.50$ & $21.13 \pm 0.08$ & $19.05 \pm 0.05$ & $16.49 \pm 0.11$ & $15.77 \pm 0.13$ & $15.33 \pm 0.14$ & $0.08 \pm 0.07$ & $228 \pm 54$ & L2.5 \\
\hline SDSS J155151.04+174216.7 & $23.34 \pm 0.24$ & $21.10 \pm 0.06$ & $19.08 \pm 0.06$ & $16.70 \pm 0.14$ & $15.83 \pm 0.15$ & $15.13 \pm 0.12$ & $0.12 \pm 0.05$ & $320 \pm 24$ & L1.5 \\
\hline SDSS J155702.82+121258.1 & $23.84 \pm 0.55$ & $21.25 \pm 0.10$ & $19.19 \pm 0.08$ & $17.12 \pm 0.25$ & $16.02 \pm 0.19$ & $15.51 \pm 0.26$ & $0.62 \pm 0.05$ & $243 \pm 4$ & L0.5 \\
\hline SDSS J160022.86+484132.8 & $23.53 \pm 0.44$ & $21.20 \pm 0.10$ & $19.06 \pm 0.05$ & $16.27 \pm 0.09$ & $15.43 \pm 0.12$ & $15.01 \pm 0.14$ & $0.46 \pm 0.09$ & $102 \pm 11$ & L4.5 \\
\hline SDSS J160835.64+120226.8 & $23.22 \pm 0.26$ & $20.56 \pm 0.04$ & $18.56 \pm 0.03$ & $16.37 \pm 0.12$ & $15.35 \pm 0.11$ & $15.00 \pm 0.14$ & $0.07 \pm 0.03$ & $301 \pm 28$ & L0.5 \\
\hline SDSS J160911.45+211658.7 & $24.02 \pm 0.64$ & $21.53 \pm 0.14$ & $19.50 \pm 0.08$ & $16.96 \pm 0.21$ & $15.97 \pm 0.20$ & $14.87 \pm 0.11$ & $0.09 \pm 0.03$ & $30 \pm 22$ & L2 \\
\hline
\end{tabular}


Table 7. continued.

\begin{tabular}{|c|c|c|c|c|c|c|c|c|c|}
\hline SDSS Name & SDSS $r$ & SDSS $i$ & $\operatorname{SDSS} z$ & 2MASS $J$ & 2MASS $H$ & 2MASS $K$ & $\begin{array}{l}\text { Proper motion } \\
\left({ }^{\prime \prime} \mathrm{yr}^{-1}\right)\end{array}$ & $\begin{array}{l}\text { Proper motion } \\
\text { Angle }\end{array}$ & $\begin{array}{l}\text { Sp. Type } \\
\text { by Colors }\end{array}$ \\
\hline SDSS J161231.01+483357.5 & $24.34 \pm 0.68$ & $21.27 \pm 0.08$ & $19.17 \pm 0.06$ & $16.20 \pm 0.10$ & $15.62 \pm 0.12$ & $14.83 \pm 0.12$ & $0.11 \pm 0.06$ & $320 \pm 38$ & L5.5 \\
\hline SDSS J161459.98+400435.1 & $22.60 \pm 0.19$ & $20.95 \pm 0.08$ & $18.93 \pm 0.04$ & $16.57 \pm 0.12$ & $15.84 \pm 0.15$ & $15.01 \pm 0.12$ & $0.30 \pm 0.04$ & $296 \pm 8$ & L1 \\
\hline SDSS J161655.06+190842.8 & $23.57 \pm 0.42$ & $21.46 \pm 0.09$ & $19.45 \pm 0.07$ & $17.18 \pm 0.19$ & $16.37 \pm 0.22$ & $15.90 \pm 0.24$ & $0.06 \pm 0.05$ & $305 \pm 59$ & L0 \\
\hline SDSS J163021.84-001801.6 & $24.80 \pm 0.69$ & $22.58 \pm 0.24$ & $19.42 \pm 0.06$ & $16.25 \pm 0.12$ & $15.55 \pm 0.15$ & $15.24 \pm 0.19$ & & & L9.5 \\
\hline SDSS J164939.25+425043.7 & $24.06 \pm 0.46$ & $21.71 \pm 0.11$ & $19.48 \pm 0.07$ & $16.79 \pm 0.14$ & $15.46 \pm 0.10$ & $14.79 \pm 0.12$ & $0.18 \pm 0.05$ & $320 \pm 15$ & L5 \\
\hline SDSS J165914.44+172642.3 & $24.22 \pm 0.48$ & $21.73 \pm 0.10$ & $19.69 \pm 0.06$ & $16.69 \pm 0.16$ & $15.54 \pm 0.13$ & $15.15 \pm 0.14$ & $0.11 \pm 0.06$ & $107 \pm 32$ & L6 \\
\hline SDSS J170418.24+744315.0 & $23.26 \pm 0.30$ & $21.32 \pm 0.07$ & $19.07 \pm 0.05$ & $16.09 \pm 0.09$ & $15.14 \pm 0.09$ & $14.27 \pm 0.09$ & $0.13 \pm 0.04$ & $217 \pm 18$ & L7.5 \\
\hline SDSS J171201.36+324456.6 & $23.99 \pm 0.44$ & $21.29 \pm 0.08$ & $19.25 \pm 0.07$ & $17.14 \pm 0.17$ & $16.26 \pm 0.18$ & $15.96 \pm 0.24$ & $0.52 \pm 0.10$ & $272 \pm 11$ & L0 \\
\hline SDSS J172545.59+640501.2 & $23.90 \pm 0.81$ & $21.90 \pm 0.23$ & $19.46 \pm 0.09$ & $16.81 \pm 0.17$ & $15.89 \pm 0.17$ & $15.35 \pm 0.20$ & $0.61 \pm 0.33$ & $12 \pm 33$ & L6.5 \\
\hline SDSS J172746.49+572247.6 & $22.53 \pm 0.21$ & $20.21 \pm 0.04$ & $18.21 \pm 0.03$ & $15.83 \pm 0.08$ & $14.96 \pm 0.09$ & $14.69 \pm 0.11$ & $0.14 \pm 0.10$ & $33 \pm 51$ & $\mathrm{~L} 1.5$ \\
\hline SDSS J210515.30-003701.5 & $24.48 \pm 0.60$ & $21.33 \pm 0.08$ & $19.10 \pm 0.05$ & $16.97 \pm 0.17$ & $15.90 \pm 0.17$ & $14.91 \pm 0.13$ & $0.07 \pm 0.07$ & $81 \pm 64$ & L1.5 \\
\hline
\end{tabular}

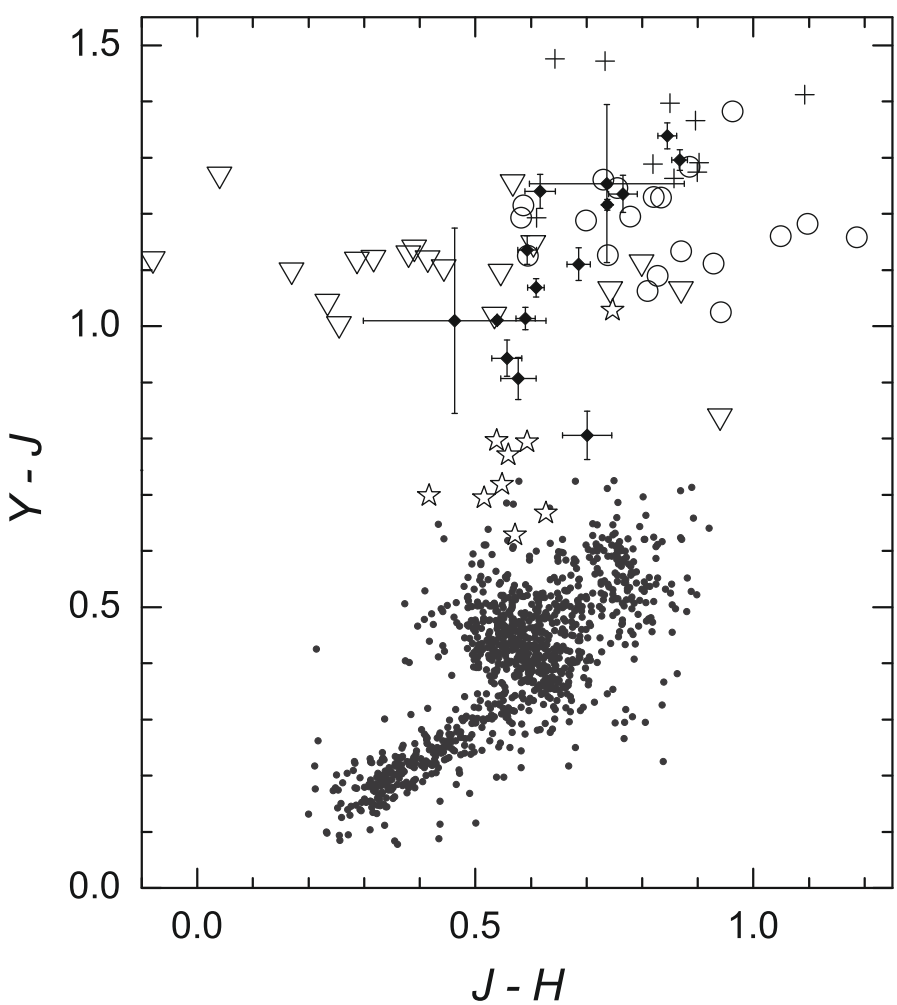

Fig. 7. $Y-J$ vs. $J-H$ diagram for known $\mathrm{M}, \mathrm{L}$ and $\mathrm{T}$ dwarfs and $15 \mathrm{ul}-$ tracool dwarf candidates (diamonds) which have $Y$ band photometric data from UKIDSS, include 4 objects with SDSS spectra. M5.5-M8.5 (open pentacles), L0-L4.5 (crosses), L5-L9.5 (open circles), T0-T3.5 (open triangles) and 1024 sources from UKIDSS LAS in $1 \mathrm{deg}^{2}$ with $Y<18.5$ (gray points). The $J$ band photometric data of the two with the very large errors used here are transformed from 2MASS.

and are more likely to become $i$-band drop outs, precluding their selection in our sample. For our full sample, we reach fainter magnitudes and thus identify more later L dwarfs. The spectral type distribution of the candidates without spectra (i.e. based on the relationship between spectral type and colors) spans a range out to T3, with many candidates in the L0-L7 range.

Our spectral typing procedure makes use of numerous optical, near infrared and optical-infrared colors. Overall we find that the $i-z, i-J, i-H$ and $i-K$ colors are the most useful. For the objects in Table 1, the spectral types based on SDSS spectra and those based on colors generally agree with each other well. However, we caution against the use of $i-z$ for estimating spectral types earlier than L3, as Fig. 3 shows $i-z$ does not correlate

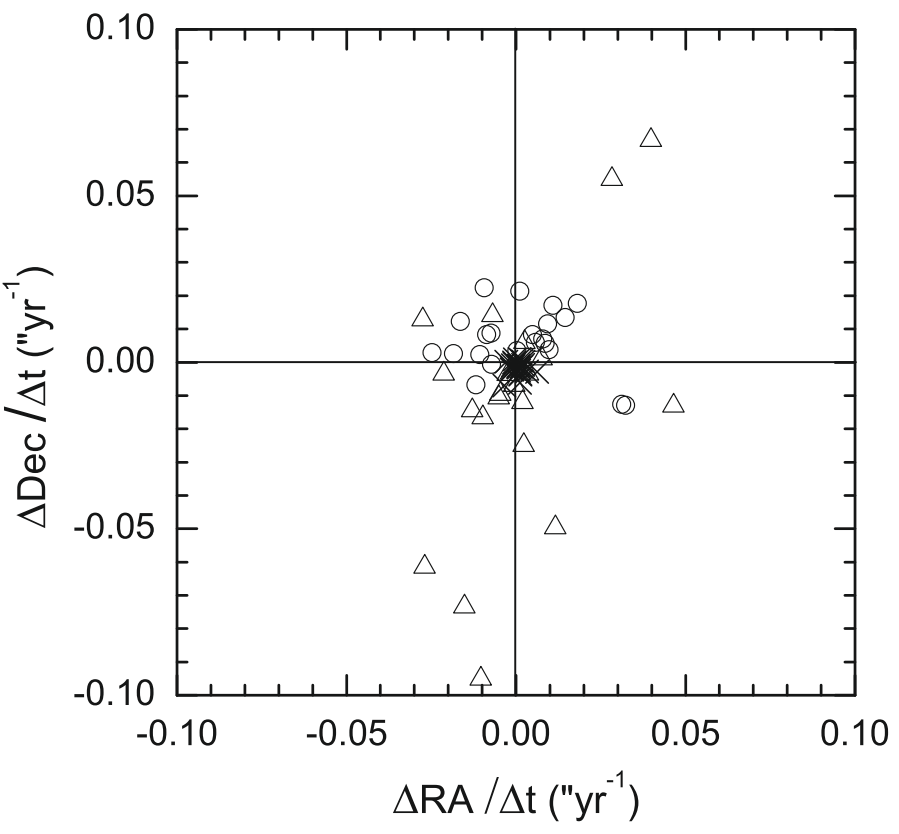

Fig. 8. Average proper motions of reference objects for ultracool dwarf candidates in Table 6. Different symbols indicated systematic coorections used for different survey combinations, 2MASS-SDSS (open triangles, 21 objects), SDSS-UKIDSS (open circles, 23 objects) and 2MASS-UKIDSS (crosses, 23 objects).

well in this range. In our analysis, if the $i$ - $J$ and $i$ - $H$ colors indicate a spectral type earlier than L3, then we do not include an estimated type based on the $i-z$ color. The addition of UKIDSS photometry adds an additional means to constrain spectral type (e.g. using $Y-J$ ), particularly in and around the L-T transition ( $\sim$ L7-T3). With the increasing coverage of UKIDSS we can refine our selection techniques through additional color-spectral type relationships in the near future.

Of the 36 objects with SDSS spectra, 19 have $2 \sigma$ detections of non-zero proper motions from SDSS-2MASS, 10 of which have proper motions above $0.2^{\prime \prime} \mathrm{yr}^{-1}$ (see, Table 1). There are fewer $2 \sigma$ proper motion detections for objects without spectra because they are, on average, further away. For SDSS-2MASS match, a matching radius of $6^{\prime \prime}$ might lead to the loss of a small number hight proper motion objects (e.g. proper motion larger than $1^{\prime \prime} \mathrm{yr}^{-1}$ and baseline longer than 6 years). Some objects in Tables 1, 6 and 7 have larger proper motions (also with large errors) but usually have a shorter baseline (even less than a year) and these proper motions are not very reliable. The errors in our proper motion measurements are dominated by 2 MASS 
positional uncertainties (especially for objects with shorter baselines), however we have shown through a variety of comparisons that our 2MASS-SDSS database proper motions are of reasonable quality and can thus provide an additional tool to identify large samples of L dwarfs in the SDSS sky. In the future, a SDSS second epoch and surveys such as Pan-STARRS will offer an even more powerful means to efficiently select late $M$ and $L$ dwarfs through their proper motion.

It is clear that SDSS combined with 2MASS and now UKIDSS, offers a powerful means to select large populations of L dwarfs using spectroscopy, photometry and astrometry. As the sample of known L dwarfs grows we can expect to reveal a broader range of inherent properties (e.g. composition, mass, age, kinematics). Higher signal-to-noise and resolution spectroscopic observations could be used to study such interesting subpopulations e.g. by searching for the presence of lithium to directly assess age and mass (Pavlenko et al. 2007) and the use of higher resolution cross correlation techniques to measure radial velocities and space motions, yielding important kinematic information.

Acknowledgements. Funding for the SDSS and SDSS-II has been provided by the Alfred P. Sloan Foundation, the Participating Institutions, the National Science Foundation, the U.S. Department of Energy, the National Aeronautics and Space Administration, the Japanese Monbukagakusho, the Max Planck Society, and the Higher Education Funding Council for England. The SDSS Web Site is http://wwW.sdss.org/. The SDSS is managed by the Astrophysical Research Consortium for the Participating Institutions. The Participating Institutions are the American Museum of Natural History, Astrophysical Institute Potsdam, University of Basel, University of Cambridge, Case Western Reserve University, University of Chicago, Drexel University, Fermilab, the Institute for Advanced Study, the Japan Participation Group, Johns Hopkins University, the Joint Institute for Nuclear Astrophysics, the Kavli Institute for Particle Astrophysics and Cosmology, the Korean Scientist Group, the Chinese Academy of Sciences (LAMOST), Los Alamos National Laboratory, the Max-Planck-Institute for Astronomy (MPIA), the Max-Planck-Institute for Astrophysics (MPA), New Mexico State University, Ohio State University, University of Pittsburgh, University of Portsmouth, Princeton University, the United States Naval Observatory, and the University of Washington. This work is based in part on data obtained as part of the UKIRT Infrared Deep Sky Survey. This publication makes use of data products from the Two Micron All Sky Survey. This research has made use of the VizieR catalogue access tool, CDS, Strasbourg, France. Research has benefitted from the M, L, and $\mathrm{T}$ dwarf compendium housed at DwarfArchives.org and maintained by Chris Gelino, Davy Kirkpatrick, and Adam Burgasser. This work was part supported by the Natural Science Foundation of China under Grant Nos. 10521001, 10433030, 10503010 and the CAS Research Fellowship for International Young Researchers.

\section{References}

Adelman-McCarthy, J. K., Aguros, M. A., Allam, S. S., et al. 2007, ApJS, 172, 634

Adelman-McCarthy, J. K., Aguros, M. A., Allam, S. S., et al. 2008, ApJS, 175, 297

Baraffe, I., Chabrier, G., Baraman, T. S., et al. 2003, A\&A, 402, 701

Becklin, E. E., \& Zuckerman, B. 1988, Nature, 336, 656

Bessell, M. S. 1991, AJ, 101, 662

Bochanski, J. J., West, A. A., Hawley, S. L., \& Covey, K. R. 2007, AJ, 133, 531

Burgasser, A. J., Kirkpatrick, J. D., Brown, M. E., et al. 1999, ApJ, 522, L65

Burgasser, A. J., Kirkpatrick, J. D., Brown, M. E., et al. 2002, ApJ, 564, 421

Burgasser, A. J., McElwain, M. W., Kirkpatrick, J. D., et al. 2004, AJ, 127, 2856 Burningham, B., Pinfield, D. J., Leggett, S. K., et al. 2008, MNRAS, accepted

Chiu, K., Fan, X., Leggett, S. K., et al. 2006, AJ, 131, 2722

Cruz, K. L., Reid, I. N., Liebert, J., Kirkpatrick, J. D., \& Lowrance, P. J. 2003, AJ, 126, 2421

Cruz, K. L., Reid, I. N., Kirkpatrick, J. D., et al. 2007, AJ, 133, 439

Delfosse, X., Tinney, C. G., Forveille, T., et al. 1997, A\&A, 327, L25

Epchtein, N., de Batz, B., Capoani, L., et al. 1997, The ESO Messeager, 87, 27

Fan, X., Knapp, G. R., Strauss, M. A., et al. 2000, AJ, 119, 928

Geballe, T. R., Knapp, G. R., Leggett, S. K., et al. 2002, ApJ, 564, 466

Gizis, J. E., Monet, D. G., Reid, I. N., et al. 2000, AJ, 120, 1085

Hawley, S. L., Covey, K. R., Knapp, G. R., et al. 2002, AJ, 123, 3409

Hewett, P. C., Warren, S. J., Leggett, S. K., \& Hodgkin, S. T. 2006, MNRAS, 367,454

Kendall, T. R., Mauron, N., Azzopardi, M., et al. 2003, A\&A, 403, 929

Kendall, T. R., Jones, H. R. A., Pinfield, D. J., et al. 2007a, MNRAS, 374, 445

Kendall, T. R., Tamura, M., Tinney, C. G., et al. 2007b, A\&A, 466, 1059

Kirkpatrick, J. D., Reid, I. N., Liebert, J., et al. 1999, ApJ, 519, 802

Kirkpatrick, J. D., Reid, I. N., Liebert, J., et al. 2000, AJ, 120, 447

Knapp, G. R., Leggett, S. K., Fan, X., et al. 2004, AJ, 127, 3553

Lawrence, A., Warren, S. J., Almaini, O., et al. 2007, MNRAS, 379, 1599

Lodieu, N., Pinfield, D. J., Leggett, S. K., et al. 2007, MNRAS, 379, 1423

Looper, D. L., Kirkpatrick, J. D., \& Burgasser, A. J. 2007, AJ, 134, 1162

Nakajima, T., Oppenheimer, B. R., Kulkarni, S. R., et al. 1995, Nature, 378, 463

Pavlenko, Ya. V., Jones, H. R. A., Martín, E. L., et al. 2007, MNRAS, 380, 1285

Pinfield, D. J., Burningham, B., Tamura, M., et al. 2008, MNRAS, 390, 304

Reid, I. N., Cruz, K. L., Kirkpatrick, J. D., et al. 2008, AJ, 136, 1290

Schneider, D. P., Knapp, G. R., Hawley, S. L., et al. 2002, AJ, 123, 458

Schmidt, S. J., Cruz, K. L., Bongiorno, B. J., Liebert, J., \& Reid, I. N. 2007, AJ, 133,2258

Skrutskie, M. F., Cutri, R. M., Stiening, R., et al. 2006, AJ, 131, 1163

Stoughton, C., Lupton, R. H., Bernardi, M., et al. 2002, AJ, 123, 485

Warren, S. J., Mortlock, D. J., Leggett, S. K., et al. 2007, MNRAS, 381, 1400

York, D. G., Adelman, J., Anderson, J. E., Jr., et al. 2000, AJ, 120, 1579 